\title{
Medicinal Plants Used to Manage Human and Livestock Ailments in Raya Kobo District of Amhara Regional State, Ethiopia
}

\author{
Ashenafi Osman, ${ }^{1}$ Desta Berhe Sbhatu $\mathbb{D D}^{2},{ }^{2}$ and Mirutse Giday ${ }^{3}$ \\ ${ }^{1}$ College of Natural and Computational Sciences, Mekelle University, P.O. Box 231, Mekelle, Ethiopia \\ ${ }^{2}$ Mekelle Institute of Technology, Mekelle University, P.O. Box 1632, Mekelle, Ethiopia \\ ${ }^{3}$ Aklilu Lemma Institute of Pathobiology, Addis Ababa University, Addis Ababa, Ethiopia
}

Correspondence should be addressed to Desta Berhe Sbhatu; desta.sbhatu@mu.edu.et

Received 13 June 2020; Revised 24 September 2020; Accepted 21 October 2020; Published 30 October 2020

Academic Editor: Tadaaki Satou

Copyright (c) 2020 Ashenafi Osman et al. This is an open access article distributed under the Creative Commons Attribution License, which permits unrestricted use, distribution, and reproduction in any medium, provided the original work is properly cited.

\begin{abstract}
Plant-based traditional medicine is practiced in Raya Kobo district, Amhara Regional State, Northeastern Ethiopia, to manage different human and livestock ailments. However, the formal ethnobotanical survey that documented such knowledge is lacking. Therefore, the aim of this study was to document the traditional knowledge on the use of medicinal plants to manage human and livestock ailments in the district. The study was conducted from January to July 2017 in five purposefully selected kebeles of the district. Ethnobotanical data were collected mainly using semistructured interviews conducted with 150 informants. In the five kebeles, 30 informants (15 males and 15 females) were selected using the stratified random sampling method from a list of traditional practitioners and knowledgeable individuals. Data were analyzed by employing descriptive and inferential statistical methods. The study documented a total of 91 medicinal plant species (distributed in 51 families) used in managing 38 human and 12 livestock ailments. Out of the total recorded plants species, 74 and 17 were used in managing human and livestock ailments, respectively. Leaves were the most frequently used plant parts in the preparation of remedies, accounting for $53.1 \%$ of the total preparations. The three most common methods of remedy preparation were grinding/pounding (23.5\%), crushing (19.8\%), and boiling (14.5\%). Preference ranking conducted by selected informants on eight medicinal plants used in treating human febrile illness locally called "mich" revealed that Ocimum urticifolium is the most preferred medicinal plant-an indication of its high potency against the disease, and therefore needs to be prioritized for future scientific investigation. The result of this study demonstrated the rich traditional knowledge and practices in the district on the use of medicinal plants in treating various human and livestock ailments. Deforestation and drought were reported to be the major factors in the district threatening the medicinal plants and the associated knowledge. Thus, concerted efforts have to be made to conserve this important heritage using every possible means.
\end{abstract}

\section{Background}

Estimates show that $80 \%$ of the population living in developing countries depends on traditional medicine for its healthcare needs [1] - a practice that largely relies on the use of plants. The high prevalence in the use of traditional medicine is mainly attributed to its low cost, efficacy, and better accessibility. Traditional medicine is also serving as a source of knowledge in the development of many plant- based synthetic drugs-e.g., morphine used as analgesic is synthesized from Papaver somniferum, aspirin used as analgesic is synthesized from Filipendula ulmaria, and quinine used in treating malaria is synthesized from Cinchona pubescens [2].

In Ethiopia, the knowledge and utilization of traditional medicine, in general, and medicinal plants, in particular, are believed to be wide due to high diversity of higher plants, estimated to reach 6,000 species [3], and rich cultures, belief 
systems, and languages. One report indicated that about $80 \%$ of the Ethiopian population is still dependent on traditional medicine principally using plants [4]. Abebe and Ayehu [5] reported the application of more than 800 medicinal plant species in the Ethiopian traditional medicinal system. Traditional medicine is the most affordable and easily accessible source of treatment in the primary healthcare system of many communities of the country. But the rich knowledge of traditional medicine that has been developed over thousands of years is being exposed to serious depletion mainly due to deforestation, environmental degradation, overexploitation, agricultural land expansion, acculturation, and limited practice of its documentation.

In Ethiopia, cognizant of the role of traditional medicine and medicinal plants and the existing threats to the associated knowledge and practices, attempts have been made, mainly in the last four decades, to document medicinal plants used by many communities. Some of the notable works include that of Giday et al. [6] (in the northwestern part), Giday and Ameni [7] (in the northern part), Gedif and Hahn [8], and Teklehaymanot et al. [9] (in the central part), Wondimu et al. [10] (in the southeast part), and Abbink [11] (in the southwestern part). However, such works are not inclusive; thus, concerted efforts should be made in the country to document such valuable knowledge for better utilization and conservation.

A number of ethnobotanical studies have been conducted in different districts of the Amhara State of Ethiopia to which the Raya Kobo district belongs [6, 12-29]. There are 11 rural districts in the North Wollo zone of the Amhara State including Raya Kobo. However, the survey of published works shows that ethnobotanical studies on medicinal plants were conducted in only 2 of the 11 districts in the zone, namely, in Guba Lafto and Delanta. The studies in Guba Lafto [26] and Delanta [24] districts reported 135 and 133 medicinal plant species, respectively. People in the Raya Kobo district, like in other communities in Ethiopia, are expected to have been heavily dependent on herbal medicine to manage human and livestock ailments. However, an ethnobotanical study that aims to document the local use of medicinal plants is lacking. Thus, the aim of this study was to document the local knowledge on the use of medicinal plants in treating human and livestock ailments.

\section{Materials and Methods}

2.1. Description of the Study Area. The study was carried out in Raya Kobo district of North Wollo zone of the Amhara State of Ethiopia (Figure 1). It is located between $11^{\bigcirc_{5}} 4^{\prime} 04^{\prime \prime}$ and $12^{\circ} 02^{\prime} 56^{\prime \prime} \mathrm{N}$ latitudes and $39^{\circ} 25^{\prime} 56^{\prime \prime}$ and $39^{\circ} 49^{\prime} 04^{\prime \prime} \mathrm{E}$ longitudes. Kobo is the administrative town of the district and is located at $570 \mathrm{~km}$ north of Addis Ababa. Raya Kobo is bordered by Raya Alamata district of Tigrai State in the north, by Guba Lafto and Gidan districts of Amhara State in the south and west, respectively, and by Golina district of Afar State in the east. The district covers a total area of $183,697.50$ hectares of land, of which $59 \%$ is kola (lowland), $38 \%$ is woyinadega (semihighland), and $3 \%$ is dega (highland) with annual temperature ranging from a minimum average of $12.31^{\circ} \mathrm{C}$ to a maximum average of $33.07^{\circ} \mathrm{C}$ and annual rainfall ranging from $500 \mathrm{~mm}$ to $800 \mathrm{~mm}$ [30]. The district is divided into 44 rural and four urban kebeles (subdistricts). Kebele is the smallest administrative unit in Ethiopia.

Raya Kobo district has a total population of 261,897 (females 128,157; males 133,740) [31]. Amharic is the mother tongue language of the great majority of the people of the district [32]. According to a recent local government data, $28 \%$ of the district is barren land, $23.7 \%$ is agricultural/ cultivated land, $24.3 \%$ is grazing land, and $15.9 \%$ is covered by shrubs and bushes. Agriculture (crop and livestock production) is the livelihood activity of the great majority of people. Crops including sorghum, teff, and pulses are the most commonly cultivated ones. Likewise, it has high populations of sheep $(201,753)$, chickens $(183,261)$, and cattle $(158,370)$. Furthermore, it is home to 47,951 goats, 22,232 donkeys, 13,864 camels, 552 mules, and 48 horses [33].

It was reported that there are seven health centers and 43 health posts in Raya Kobo. Malaria, common cold, tuberculosis, mich (febrile illness), pneumonia, HIV-AIDS, nekersa (chronic wound), hepatitis, diarrhea, typhoid fevertyphus, and diabetics are the top ten diseases of major public health priorities in the district [34]. Unpublished 2019 government data revealed that blackleg, anthrax, pasteurellosis, sheep and goat pox, lumpy skin disease, mange, tick infestation, and internal parasitic infections are the top eight diseases of veterinary health priorities. But, only 24 veterinary clinics were operating in 2019 [33].

2.2. Selection of Study Kebeles and Informants. Five kebeles, namely, Menjelo, Bewa, Gedeba, Ayub-Amaya, and Mendeferana Golesha (Figure 1), were purposively selected for the study following the approach of Martin [35] with the assistance of district authorities, elders, and knowledgeable persons based on the availability of traditional practitioners and knowledgeable individuals. For this purpose, many individuals, aged 40 years and above who claimed to be practitioners of traditional medicine and believed to be knowledgeable, were identified from each of the five kebeles. Then, 30 informants ( 15 males and 15 females) were selected from each kebele using a stratified random sampling approach [36].

2.3. Collection of Ethnobotanical Data. Ethnobotanical data were collected between January and July 2017 mainly through (a) semistructured interviews conducted with the sampled informants using a list of questions prepared beforehand in Amharic, mother tongue language in the study district, and (b) field observations using the procedures recommended by Martin [35] and Alexiades [37]. Gathered data and information were local names of medicinal plants, plant part(s) utilized, method of preparation, ailments treated or prevented, and routes of administration. Specimens of most of the medicinal plants were collected and identified by botanists at the Ethiopian National Herbarium (ETH) and Aklilu Lemma Institute of Pathobiology 


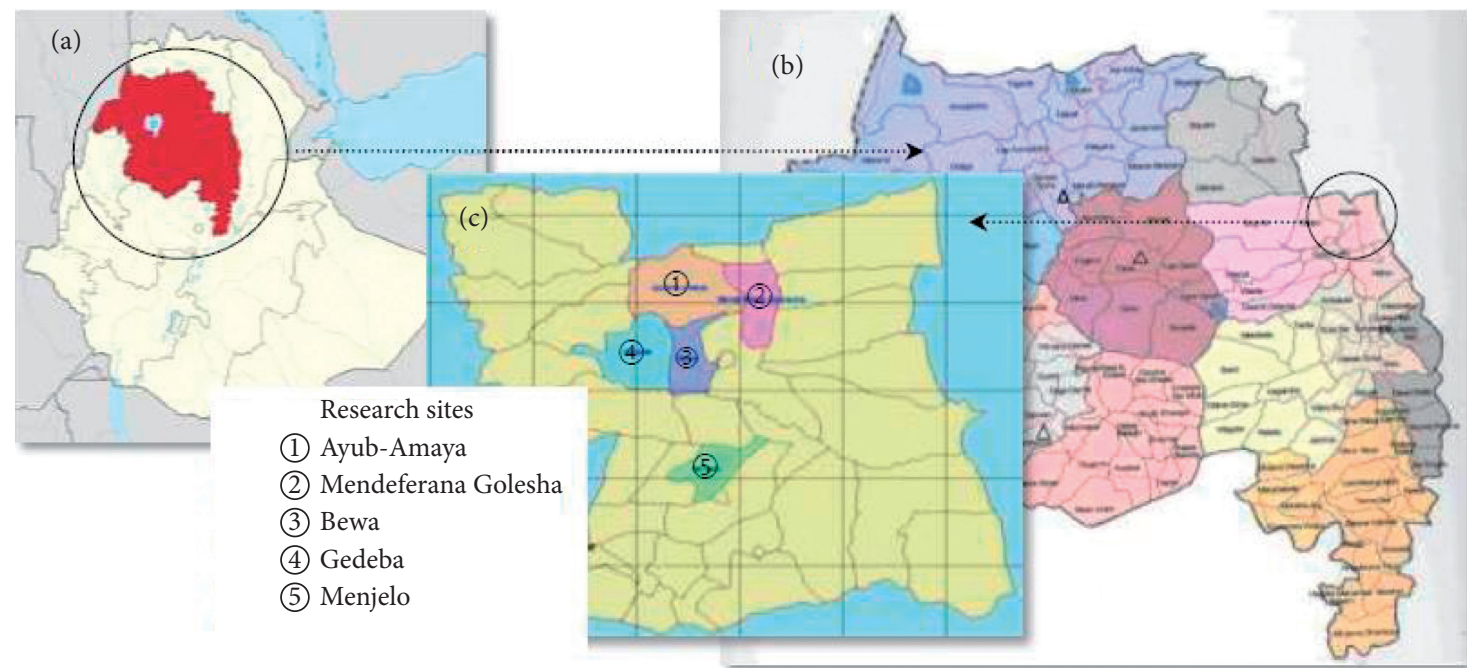

Figure 1: Research site: (a) Ethiopia, (b) Amhara State, and (c) Raya Kobo district (not legal map).

(ALIPB), Addis Ababa University, Ethiopia, and vouchers were deposited at the Endod and Other Medicinal Plants Research Unit, ALIPB.

2.4. Simple Preference Ranking. Preference ranking was performed by seven informants on eight medicinal plants used in treating mich (a human febrile illness) following the guideline established by Martin [35]. Informants for this exercise were randomly selected from those individuals already involved in semistructured interviews. The eight plants had the highest informant consensus among a total of 13 plants reported to be used in treating mich. Of all the ailments reported, mich was found to be treated by the highest number of medicinal plants-an indication of the high health importance of the ailment in the district. The highest value (i.e., 8) was given to medicinal plants considered to be the most effective, and the least value (i.e., 1) was given to the plant considered as the least effective.

2.5. Data Analysis. Descriptive statistical methods were used to produce frequencies and percentages. Coefficient of correlation and analysis of variance (ANOVA) were used to compare mean values of knowledge of medicinal plants among different groups of informants.

\section{Results and Discussion}

3.1. Diversity of Medicinal Plants. The present study documented a total of 91 species of medicinal plants. Whereas 74 of the species were used to manage human health problems only (Table 1), and 17 of them were used against livestock ailments only (Table 2). Some examples are given in Figure 2. As reflected by the richness of the plants used and the diversity of ailments treated, the results showed the profound roles of medicinal plants in meeting the basic healthcare needs of the communities in the study area. Previous studies carried out in Delanta and Guba Lafto districts of North Wollo zone, to which the study district belongs, reported the use of 135 and 133 species of medicinal plants, respectively, to manage various ailments $[24,26]$. Of the reported medicinal plants, $42(46.2 \%)$ were herbs, $29(31.8 \%)$ were shrubs, and 20 (22\%) were trees. Previous ethnobotanical studies conducted elsewhere in several districts of Ethiopia also revealed the wide use of herbs as medicinal plants $[14,38-40]$. Such wide use of herbs in the study district may be attributed to their ease of harvesting and processing and their relatively better abundance compared to shrubs and trees as it was observed by the investigators and documented in the local government records [33].

The reported medicinal plants belonged to 51 families and 83 genera. Whereas six species belonged to Fabaceae, five species belonged to each of the families of Asteraceae, Euphorbiaceae, and Lamiaceae. Solanaceae was represented by four species, while Boraginaceae, Cucurbitaceae, Ranunculaceae, and Vitaceae were represented by three species each. Each of the remaining 42 families was represented by two or one species. The fact that Fabaceae, Asteraceae, Euphorbiaceae, and Lamiaceae contributed higher number of medicinal plants could probably be attributed to their better species richness in the flora of Ethiopia. Fabaceae, Asteraceae, Euphorbiaceae, and Lamiaceae are among the largest families in the flora of Ethiopia and Eritrea containing 486, 440, 209, and 184 species, respectively [41-44].

3.2. Ailments Managed by Medicinal Plants. The medicinal plants documented by the present study were used for treating 38 human ailments (Table 3) and 12 livestock diseases (Table 4). Of the 74 medicinal plants used for treating human ailments, 17,13 , and 10 were used to treat gastrointestinal complaints, mich, and wound, respectively; while snake bite and devil's illness were found to be treated by six medicinal plants each. Each of the remaining 33 human ailments was reported to be managed by four or fewer medicinal plants (Table 3). Diarrhea, mich, and wound were among the top ten diseases of major public health 
TABLE 1: Medicinal plants used to treat human ailments.

\begin{tabular}{|c|c|c|c|c|c|c|c|c|}
\hline Scientific name & Local name & $\begin{array}{l}\text { Voucher } \\
\text { number }\end{array}$ & Habit & $\begin{array}{l}\text { Ailment } \\
\text { treated }\end{array}$ & $\begin{array}{c}\text { Plant part } \\
\text { used }\end{array}$ & $\begin{array}{c}\text { Preparation } \\
\text { methods }\end{array}$ & $\begin{array}{l}\text { Administration } \\
\text { route }\end{array}$ & $\begin{array}{c}\text { No. of } \\
\text { informants }\end{array}$ \\
\hline \multirow[t]{2}{*}{$\begin{array}{l}\text { Acacia abyssinica } \\
\text { Hochst. ex Benth. } \\
\text { (Fabaceae) }\end{array}$} & Grar & $\begin{array}{l}\text { AS-73- } \\
2017\end{array}$ & Tree & Allergy & Leaf & $\begin{array}{l}\text { Pounding and then } \\
\text { squeezing }\end{array}$ & Dermal & 11 \\
\hline & & & & Broken bone & Root & Crushing & $\begin{array}{l}\text { Dermal on the } \\
\text { broken bone }\end{array}$ & 15 \\
\hline $\begin{array}{l}\text { Acokanthera schimperi } \\
\text { (A.DC.) Schweinf. } \\
\text { (Apocynaceae) }\end{array}$ & Merez & $\begin{array}{l}\text { AS-69- } \\
2017\end{array}$ & Tree & Itch & Stem bark & $\begin{array}{l}\text { Drying and then } \\
\text { grinding }\end{array}$ & Dermal & 12 \\
\hline $\begin{array}{l}\text { Allium cepa } \\
\text { L. (Alliaceae) }\end{array}$ & Key shinkurt & $\begin{array}{l}\text { AS-78- } \\
2017\end{array}$ & Herb & $\begin{array}{l}\text { Stomach } \\
\text { complaints }\end{array}$ & $\begin{array}{l}\text { Bulb } \\
\text { (leaf) }\end{array}$ & $\begin{array}{c}\text { Pounding after } \\
\text { mixing it with } \\
\text { A. sativum and } \\
\text { Ruta chalepensis, } \\
\text { and then adding } \\
\text { honey }\end{array}$ & Oral & 7 \\
\hline \multirow[t]{6}{*}{$\begin{array}{l}\text { Allium sativum } \\
\text { L. (Alliaceae) }\end{array}$} & $\begin{array}{c}\text { Nech } \\
\text { shinkurt }\end{array}$ & - & Herb & $\begin{array}{l}\text { Cough } \\
\text { Stomach } \\
\text { complaints }\end{array}$ & $\begin{array}{l}\text { Bulb } \\
\text { (leaf) }\end{array}$ & $\begin{array}{l}\text { Pounding or } \\
\text { chewing }\end{array}$ & Oral & $\begin{array}{c}8 \\
10\end{array}$ \\
\hline & & & & Headache & $\begin{array}{l}\text { Bulb } \\
\text { (leaf) }\end{array}$ & $\begin{array}{l}\text { Pounding and } \\
\text { chewing }\end{array}$ & Oral & 11 \\
\hline & & & & Malaria & $\begin{array}{l}\text { Bulb } \\
\text { (leaf) }\end{array}$ & $\begin{array}{l}\text { Pounding after } \\
\text { mixing it with Cicer } \\
\text { arietinum }\end{array}$ & Oral & 5 \\
\hline & & & & Cough & $\begin{array}{l}\text { Bulb } \\
\text { (leaf) }\end{array}$ & $\begin{array}{l}\text { Pounding or } \\
\text { crushing and then } \\
\text { mixing it with } \\
\text { honey }\end{array}$ & Oral & 14 \\
\hline & & & & Amoeba & $\begin{array}{l}\text { Bulb } \\
\text { (leaf) }\end{array}$ & $\begin{array}{l}\text { Grinding and then } \\
\text { mixing it with } \\
\text { honey }\end{array}$ & Oral & 6 \\
\hline & & & & $\begin{array}{l}\text { Lung cancer/ } \\
\text { tuberculosis }\end{array}$ & $\begin{array}{l}\text { Bulb } \\
\text { (leaf) }\end{array}$ & $\begin{array}{l}\text { Grinding and then } \\
\text { mixing it with } \\
\text { honey }\end{array}$ & Oral & 4 \\
\hline \multirow[t]{3}{*}{ Aloe spp.(Aloaceae) } & Eret & $\begin{array}{l}\text { AS-29- } \\
2017\end{array}$ & Herb & Wound & Stem & $\begin{array}{l}\text { Cutting to harvest } \\
\text { the jelly juice }\end{array}$ & Dermal & 16 \\
\hline & & & & Malaria & Stem & $\begin{array}{l}\text { Cutting to harvest } \\
\text { the jelly juice }\end{array}$ & Oral & 12 \\
\hline & & & & Diarrhea & Root & $\begin{array}{l}\text { Cutting to harvest } \\
\text { the jelly juice }\end{array}$ & Oral & 13 \\
\hline $\begin{array}{l}\text { Alternanthera pungens } \\
\text { Kunth } \\
\text { (Amaranthaceae) }\end{array}$ & $\begin{array}{l}\text { Yemeret } \\
\text { Kitigne/get }\end{array}$ & $\begin{array}{c}\text { AS-58- } \\
2017\end{array}$ & Herb & Wound & Leaf & $\begin{array}{l}\text { Grinding or } \\
\text { pounding }\end{array}$ & $\begin{array}{l}\text { Dermal on the } \\
\text { wounded part }\end{array}$ & 6 \\
\hline $\begin{array}{l}\text { Argemone mexicana } \\
\text { L. (Papaveraceae) }\end{array}$ & Enkushashle & $\begin{array}{l}\text { AS-57- } \\
2017\end{array}$ & Herb & Sore & Stem & $\begin{array}{l}\text { Cutting to harvest } \\
\text { the latex }\end{array}$ & $\begin{array}{l}\text { Dermal on the } \\
\text { affected part }\end{array}$ & 11 \\
\hline $\begin{array}{l}\text { Artemisia absinthium } \\
\text { L. (Asteraceae) }\end{array}$ & Natra & $\begin{array}{l}\text { AS-22- } \\
2017\end{array}$ & Herb & $\begin{array}{l}\text { Uvula } \\
\text { infection }\end{array}$ & $\begin{array}{l}\text { Whole } \\
\text { plant }\end{array}$ & $\begin{array}{l}\text { Squeezing and } \\
\text { producing juice }\end{array}$ & Oral & 4 \\
\hline $\begin{array}{l}\text { Azadirachta indica } \\
\text { A. Juss. (Meliaceae) }\end{array}$ & Nim & $\begin{array}{l}\text { AS-41- } \\
2017\end{array}$ & Tree & Cough & Leaf & $\begin{array}{l}\text { Boiling in water } \\
\text { after mixing it with } \\
\text { Eucalyptus globulus }\end{array}$ & $\begin{array}{c}\text { Inhalation (oral } \\
\text { and nasal) }\end{array}$ & 10 \\
\hline \multirow[t]{3}{*}{$\begin{array}{l}\text { Balanites aegyptiaca } \\
\text { (L.) Delile } \\
\text { (Zygophyllaceae) }\end{array}$} & Bedena & $\begin{array}{l}\text { AS-61- } \\
2017\end{array}$ & Tree & Tinea nigra & Leaf & Crushing & $\begin{array}{l}\text { Dermal on the } \\
\text { affected part }\end{array}$ & 5 \\
\hline & & & & $\begin{array}{l}\text { Stomach } \\
\text { complaints }\end{array}$ & Fruit & Chewing & Oral & 7 \\
\hline & & & & $\begin{array}{l}\text { Bloody } \\
\text { diarrhea }\end{array}$ & Leaf & $\begin{array}{l}\text { Crushing to collect } \\
\text { juice }\end{array}$ & Oral & 8 \\
\hline $\begin{array}{l}\text { Brassica nigra } \\
\text { L. (Brassicaceae) }\end{array}$ & Senafich & - & Herb & $\begin{array}{l}\text { Stomach } \\
\text { complaints }\end{array}$ & Seed & $\begin{array}{l}\text { Dying, then } \\
\text { grinding after } \\
\text { mixing it with } \\
\text { A. sativum and } \\
\text { Vicia faba }\end{array}$ & Oral & 6 \\
\hline
\end{tabular}


TABle 1: Continued.

\begin{tabular}{|c|c|c|c|c|c|c|c|c|}
\hline Scientific name & Local name & $\begin{array}{l}\text { Voucher } \\
\text { number }\end{array}$ & Habit & $\begin{array}{c}\text { Ailment } \\
\text { treated }\end{array}$ & $\begin{array}{c}\text { Plant part } \\
\text { used }\end{array}$ & $\begin{array}{c}\text { Preparation } \\
\text { methods }\end{array}$ & $\begin{array}{l}\text { Administration } \\
\text { route }\end{array}$ & $\begin{array}{c}\text { No. of } \\
\text { informants }\end{array}$ \\
\hline $\begin{array}{l}\text { Biancaea decapetala } \\
\text { (Roth) O. Deg. } \\
\text { (Fabaceae) }\end{array}$ & Kentefa & $\begin{array}{l}\text { AS-70- } \\
2017\end{array}$ & Shrub & Evil eye & $\begin{array}{l}\text { Leaf and } \\
\text { root }\end{array}$ & $\begin{array}{l}\text { Pounding it after } \\
\text { mixing it with } R u t a \\
\text { chalepensis }\end{array}$ & Oral & 8 \\
\hline $\begin{array}{l}\text { Calotropis procera } \\
\text { (Aiton) W.T. Aiton } \\
\text { (Apocynaceae) }\end{array}$ & Topia & $\begin{array}{l}\text { AS-48- } \\
2017\end{array}$ & Shrub & Wart & Leaf & $\begin{array}{l}\text { Cutting to harvest } \\
\text { the latex }\end{array}$ & $\begin{array}{l}\text { Dermal on the } \\
\text { affected part }\end{array}$ & 9 \\
\hline $\begin{array}{l}\text { Carica papaya } \\
\text { L. (Caricaceae) }\end{array}$ & Papaye & $\begin{array}{l}\text { AS-55- } \\
2017\end{array}$ & Herb & $\begin{array}{l}\text { Swelling on } \\
\text { the skin }\end{array}$ & $\begin{array}{l}\text { Fruit, } \\
\text { seeds }\end{array}$ & Splitting & $\begin{array}{l}\text { Dermal on the } \\
\text { swollen part }\end{array}$ & 8 \\
\hline \multirow[t]{3}{*}{$\begin{array}{l}\text { Carissa spinarum } \\
\text { L. (Apocynaceae) }\end{array}$} & Agam & $\begin{array}{l}\text { AS-30- } \\
2017\end{array}$ & Shrub & Mental stress & Root & Boiling it in water & Dermal & 3 \\
\hline & & & & Devil's illness & Leaf & $\begin{array}{l}\text { Boiling it in water } \\
\text { after mixing with } \\
\text { Croton } \\
\text { macrostachyus } \\
\text { roots }\end{array}$ & $\begin{array}{l}\text { Inhalation (oral } \\
\text { and nasal) }\end{array}$ & 7 \\
\hline & & & & Snake bite & Leaf & $\begin{array}{l}\text { Pounding after } \\
\text { mixing with leaves } \\
\text { of Verbena } \\
\text { officinalis }\end{array}$ & Oral & 5 \\
\hline $\begin{array}{l}\text { Caylusea abyssinica } \\
\text { (Fresen.) Fisch. \& C.A. } \\
\text { Mey. (Resedaceae) }\end{array}$ & Kibezelzil & $\begin{array}{l}\text { AS-64- } \\
2017\end{array}$ & Herb & Mich & Leaf & Boiling it in water & $\begin{array}{l}\text { Inhalation (oral } \\
\text { and nasal) }\end{array}$ & 15 \\
\hline \multirow{3}{*}{$\begin{array}{l}\text { Chenopodiastrum } \\
\text { murale (L.) S. Fuentes, } \\
\text { Uotila \& Borsch } \\
\text { (Amaranthaceae) } \\
\text { Citrullus lanatus } \\
\text { (Thunb.) Matsum. \& } \\
\text { Nakai (Cucurbitaceae) }\end{array}$} & Amedmado & $\begin{array}{l}\text { AS-75- } \\
2017\end{array}$ & Herb & Broken bone & Leaf & Crushing & $\begin{array}{l}\text { Dermal on the } \\
\text { broken bone }\end{array}$ & 5 \\
\hline & Habhab & - & Herb & Hypertension & Fruit & Squeezing & Oral & 4 \\
\hline & & & & $\begin{array}{l}\text { Stomach } \\
\text { complaints }\end{array}$ & Fruit & Slicing & Oral & 9 \\
\hline $\begin{array}{l}\text { Citrus limon (L.) } \\
\text { Osbeck (Rutaceae) }\end{array}$ & Lomi & $\begin{array}{c}\text { AS-59- } \\
2017\end{array}$ & Tree & Stomach ache & Fruit & $\begin{array}{l}\text { Squeezing it to } \\
\text { produce juice and } \\
\text { then adding honey }\end{array}$ & Oral & 16 \\
\hline $\begin{array}{l}\text { Clematis hirsuta Perr. } \\
\text { \& Guill. } \\
\text { (Ranunculaceae) }\end{array}$ & Chicho & $\begin{array}{l}\text { AS-67- } \\
2017\end{array}$ & Herb & Cancer & Leaf & Crushing & $\begin{array}{l}\text { Dermal on the } \\
\text { affected part }\end{array}$ & 7 \\
\hline $\begin{array}{l}\text { Clematis simensis } \\
\text { Fresen. } \\
\text { (Ranunculaceae) }\end{array}$ & Azo hareg & $\begin{array}{l}\text { AS-36- } \\
2017\end{array}$ & Herb & Cancer & Leaf & Crushing & $\begin{array}{l}\text { Dermal on the } \\
\text { affected part }\end{array}$ & 4 \\
\hline \multirow[t]{2}{*}{$\begin{array}{l}\text { Coffea arabica } \\
\text { L. (Rubiaceae) }\end{array}$} & Buna & $\begin{array}{c}\text { AS-46- } \\
2017\end{array}$ & Shrub & Wound & Seed & $\begin{array}{l}\text { Roasting and } \\
\text { pounding }\end{array}$ & $\begin{array}{l}\text { Dermal on } \\
\text { wound }\end{array}$ & 12 \\
\hline & & & & Asthma & Seed & $\begin{array}{l}\text { Roasting and } \\
\text { pounding }\end{array}$ & Oral & 6 \\
\hline \multirow[t]{2}{*}{$\begin{array}{l}\text { Cordia africana Lam. } \\
\text { (Boraginaceae) }\end{array}$} & Wanza & $\begin{array}{c}\text { AS-62- } \\
2017\end{array}$ & Tree & $\begin{array}{l}\text { Hepatitis/ } \\
\text { jaundice }\end{array}$ & Leaf & $\begin{array}{l}\text { Boiled in water } \\
\text { after mixing it with } \\
\text { Sorghum bicolor, } \\
\text { chewing }\end{array}$ & Oral & 8 \\
\hline & & & & Mich & Leaf & Boiling it in water & $\begin{array}{c}\text { Inhale (oral and } \\
\text { nasal) }\end{array}$ & 10 \\
\hline \multirow[t]{2}{*}{$\begin{array}{l}\text { Croton macrostachyus } \\
\text { Hochst. ex Delile } \\
\text { (Euphorbiaceae) }\end{array}$} & Mekenisa & $\begin{array}{c}\text { AS-19- } \\
2017\end{array}$ & Tree & Tinea nigra & Leaf & $\begin{array}{l}\text { Grinding or } \\
\text { pounding }\end{array}$ & Dermal & 4 \\
\hline & & & & Mich & Leaf & Boiling it in water & $\begin{array}{c}\text { Inhale (oral and } \\
\text { nasal) }\end{array}$ & 7 \\
\hline $\begin{array}{l}\text { Cucumis dipsaceus } \\
\text { C.G. Ehrenb. ex Spach } \\
\text { (Cucurbitaceae) }\end{array}$ & $\begin{array}{l}\text { Yemdir } \\
\text { embuay }\end{array}$ & $\begin{array}{c}\text { AS-13- } \\
2017\end{array}$ & Herb & Wound & Fruit & Crushing & Dermal & 10 \\
\hline
\end{tabular}


TABle 1: Continued.

\begin{tabular}{|c|c|c|c|c|c|c|c|c|}
\hline Scientific name & Local name & $\begin{array}{l}\text { Voucher } \\
\text { number }\end{array}$ & Habit & $\begin{array}{c}\text { Ailment } \\
\text { treated }\end{array}$ & $\begin{array}{c}\text { Plant part } \\
\text { used }\end{array}$ & $\begin{array}{c}\text { Preparation } \\
\text { methods }\end{array}$ & $\begin{array}{l}\text { Administration } \\
\text { route }\end{array}$ & $\begin{array}{c}\text { No. of } \\
\text { informants }\end{array}$ \\
\hline & & & & $\begin{array}{l}\text { Jaundice/ } \\
\text { hepatitis }\end{array}$ & $\begin{array}{l}\text { Whole } \\
\text { plant }\end{array}$ & $\begin{array}{l}\text { Chewing or } \\
\text { crushing after } \\
\text { adding water and } \\
\text { then filtering }\end{array}$ & Dermal & 4 \\
\hline \multirow[t]{2}{*}{$\begin{array}{l}\text { Cynoglossum } \\
\text { lanceolatum Forssk. } \\
\text { (Boraginaceae) }\end{array}$} & $\begin{array}{c}\text { Yegid } \\
\text { Zemedie }\end{array}$ & $\begin{array}{l}\text { AS-17- } \\
2017\end{array}$ & Herb & Mich & Leaf & Squeezing & Auricular & 12 \\
\hline & & & & $\begin{array}{l}\text { Abdominal } \\
\text { pain }\end{array}$ & Leaf & Chewing & Oral & 4 \\
\hline $\begin{array}{l}\text { Datura stramonium } \\
\text { L. (Solanaceae) }\end{array}$ & Banjie & $\begin{array}{l}\text { AS-24- } \\
2017\end{array}$ & Herb & Hemorrhoids & Leaf & Squeezing & Dermal & 6 \\
\hline $\begin{array}{l}\text { Dyschoriste radicans } \\
\text { Nees (Acanthaceae) }\end{array}$ & Telba Titi & $\begin{array}{l}\text { AS-21- } \\
2017\end{array}$ & Herb & Anthrax & $\begin{array}{l}\text { Whole } \\
\text { plant }\end{array}$ & Crushing & $\begin{array}{l}\text { Dermal on the } \\
\text { swollen part }\end{array}$ & 3 \\
\hline $\begin{array}{l}\text { Eucalyptus globulus } \\
\text { Labill. (Myrtaceae) }\end{array}$ & $\begin{array}{c}\text { Nech } \\
\text { bahirzaf }\end{array}$ & $\begin{array}{l}\text { AS-72- } \\
2017\end{array}$ & Tree & $\begin{array}{l}\text { Common } \\
\text { cold }\end{array}$ & Leaf & $\begin{array}{l}\text { Boiling in water } \\
\text { after mixing it with } \\
\text { Withania } \\
\text { somnifera }\end{array}$ & $\begin{array}{l}\text { Inhalation (oral } \\
\text { and nasal) }\end{array}$ & 14 \\
\hline & & & & Devil's illness & & & & 8 \\
\hline \multirow[t]{5}{*}{$\begin{array}{l}\text { Euclea divinorum } \\
\text { Hiern. (Ebenaceae) }\end{array}$} & Dedoho & $\begin{array}{l}\text { AS-26- } \\
2017\end{array}$ & Shrub & Mental stress & Root & Boiling in water & Oral & 3 \\
\hline & & & & Joint ache & Stem & $\begin{array}{l}\text { Putting it on fire to } \\
\text { yield smoke }\end{array}$ & $\begin{array}{l}\text { Fumigation } \\
\text { (nasal) }\end{array}$ & 8 \\
\hline & & & & Evil eye & Whole & Crushing & $\begin{array}{l}\text { Dermal around } \\
\text { the neck }\end{array}$ & 7 \\
\hline & & & & Snake bite & Root bark & $\begin{array}{l}\text { Crushing and } \\
\text { pounding after } \\
\text { adding water }\end{array}$ & Oral & 3 \\
\hline & & & & $\begin{array}{l}\text { Black spider } \\
\text { bite }\end{array}$ & Root & Chewing & Oral & 9 \\
\hline \multirow[t]{2}{*}{$\begin{array}{l}\text { Euphorbia sp. } \\
\text { (Euphorbiaceae) }\end{array}$} & Qulqualda & $\begin{array}{l}\text { AS-38- } \\
2017\end{array}$ & Shrub & $\begin{array}{l}\text { Thorn inside } \\
\text { the skin }\end{array}$ & Leaf & $\begin{array}{l}\text { Cutting to harvest } \\
\text { the latex }\end{array}$ & $\begin{array}{l}\text { Dermal on the } \\
\text { affected skin }\end{array}$ & 10 \\
\hline & & & & Cancer & Leaf & $\begin{array}{l}\text { Cutting to harvest } \\
\text { the latex }\end{array}$ & $\begin{array}{l}\text { Dermal (tying it } \\
\text { on the wound) }\end{array}$ & 3 \\
\hline $\begin{array}{l}\text { Euphorbia tirucalli } \\
\text { L. (Euphorbiaceae) }\end{array}$ & Kinchibt & $\begin{array}{l}\text { AS-53- } \\
2017\end{array}$ & Shrub & Hemorrhoids & Stem & $\begin{array}{l}\text { Cutting to harvest } \\
\text { the latex }\end{array}$ & $\begin{array}{l}\text { Dermal on the } \\
\text { affected part }\end{array}$ & 3 \\
\hline \multirow{3}{*}{$\begin{array}{l}\text { Ficus palmata Forsskål } \\
\text { (Moraceae) }\end{array}$} & & & & Wart & Stem & $\begin{array}{l}\text { Cutting to harvest } \\
\text { the latex }\end{array}$ & $\begin{array}{l}\text { Dermal on the } \\
\text { infected part }\end{array}$ & 8 \\
\hline & Beles & $\begin{array}{l}\text { AS-16- } \\
2017\end{array}$ & Shrub & Wound & Leaf & $\begin{array}{l}\text { Grinding or } \\
\text { pounding }\end{array}$ & Dermal & 9 \\
\hline & & & & Tinea nigra & Leaf & $\begin{array}{c}\text { Cutting the leaves } \\
\text { and harvesting the } \\
\text { latex }\end{array}$ & Dermal & 6 \\
\hline $\begin{array}{l}\text { Guizotia abyssinica } \\
\text { (L.f.) Cass. } \\
\text { (Asteraceae) }\end{array}$ & Nug & - & Herb & Gastritis & Seed & Pounding & Oral & 12 \\
\hline $\begin{array}{l}\text { Hagenia abyssinica } \\
\text { Willd. (Rosaceae) }\end{array}$ & Kosso & - & Tree & $\begin{array}{l}\text { Stomach } \\
\text { distention }\end{array}$ & Root & $\begin{array}{l}\text { Grinding and } \\
\text { stirring the powder } \\
\text { in tella (locally } \\
\text { produced drink) }\end{array}$ & Oral & 16 \\
\hline \multirow[t]{2}{*}{$\begin{array}{l}\text { Heliotropium } \\
\text { cinerascens A. DC. } \\
\text { (Boraginaceae) }\end{array}$} & Nechilo & $\begin{array}{l}\text { AS-39- } \\
2017\end{array}$ & Shrub & Mich & Leaf & Boiling it in water & $\begin{array}{c}\text { Inhale (oral and } \\
\text { nasal) }\end{array}$ & 15 \\
\hline & & & & Wound & Leaf & $\begin{array}{l}\text { Boiling it in water } \\
\text { after mixing it with } \\
\text { Zehneria scabra }\end{array}$ & $\begin{array}{l}\text { Dermal on the } \\
\text { affected part }\end{array}$ & 7 \\
\hline \multirow{2}{*}{$\begin{array}{l}\text { Hydnora johannis } \\
\text { Becc. (Hydnoraceae) } \\
\text { Jasminum } \\
\text { grandiflorum } \\
\text { L. (Oleaceae) }\end{array}$} & Demerech & $\begin{array}{l}\text { AS-77- } \\
2017\end{array}$ & Herb & Wound & Root & Crushing & $\begin{array}{l}\text { Dermal on the } \\
\text { wounded part }\end{array}$ & 8 \\
\hline & Tembelel & $\begin{array}{c}\text { AS-14- } \\
2017\end{array}$ & Shrub & Snake bite & Leaf & $\begin{array}{l}\text { Crushing and } \\
\text { squeezing to collect } \\
\text { juice }\end{array}$ & Oral & 6 \\
\hline
\end{tabular}


TABle 1: Continued.

\begin{tabular}{|c|c|c|c|c|c|c|c|c|}
\hline Scientific name & Local name & $\begin{array}{l}\text { Voucher } \\
\text { number }\end{array}$ & Habit & $\begin{array}{l}\text { Ailment } \\
\text { treated }\end{array}$ & $\begin{array}{c}\text { Plant part } \\
\text { used }\end{array}$ & $\begin{array}{l}\text { Preparation } \\
\text { methods }\end{array}$ & $\begin{array}{l}\text { Administration } \\
\text { route }\end{array}$ & $\begin{array}{c}\text { No. of } \\
\text { informants }\end{array}$ \\
\hline & & & & Anthrax & Leaf & $\begin{array}{l}\text { Drying and then } \\
\text { pounding }\end{array}$ & Dermal & 3 \\
\hline $\begin{array}{l}\text { Juniperus procera } \\
\text { Hochst. ex Endl. } \\
\text { (Cupressaceae) }\end{array}$ & $\begin{array}{l}\text { Yehabesha- } \\
\text { tsid }\end{array}$ & - & Herb & Cough & $\begin{array}{l}\text { Stem/ } \\
\text { root }\end{array}$ & $\begin{array}{l}\text { Crushing or } \\
\text { grinding and } \\
\text { putting it on fire or } \\
\text { boiling it in water }\end{array}$ & $\begin{array}{l}\text { Fumigation or } \\
\text { inhalation }\end{array}$ & 7 \\
\hline \multirow{3}{*}{$\begin{array}{l}\text { Justicia schimperiana } \\
\text { (Hochst. ex Nees) } \\
\text { T. Anders } \\
\text { (Acanthaceae) }\end{array}$} & Simiza & $\begin{array}{l}\text { AS-34- } \\
2017\end{array}$ & Shrub & Mental stress & Root & Boiling it in water & Dermal & 3 \\
\hline & & & & Mich & Leaf & Chewing & Oral & 11 \\
\hline & & & & Jaundice & Leaf & $\begin{array}{c}\text { Squeezing } \\
\text { Pounding it after }\end{array}$ & Oral & 6 \\
\hline $\begin{array}{l}\text { Lawsonia inermis } \\
\text { L. (Lythraceae) }\end{array}$ & Hina & $\begin{array}{l}\text { AS-54- } \\
2017\end{array}$ & Shrub & $\begin{array}{l}\text { Rheumatic } \\
\text { disease }\end{array}$ & Leaf & $\begin{array}{l}\text { mixing it with } \\
\text { Citrus limon and } \\
\text { adding water }\end{array}$ & Oral & 3 \\
\hline $\begin{array}{l}\text { Lepidium sativum } \\
\text { L. (Brassicaceae) }\end{array}$ & Feto & - & Herb & $\begin{array}{l}\text { Stomach } \\
\text { complaints }\end{array}$ & Seed & $\begin{array}{l}\text { Grinding and } \\
\text { mixing it in water }\end{array}$ & Oral & 15 \\
\hline $\begin{array}{l}\text { Leucas abyssinica } \\
\text { (Benth.) Briq. } \\
\text { (Lamiaceae) }\end{array}$ & Aftegegne & $\begin{array}{l}\text { AS-03- } \\
2017\end{array}$ & Shrub & Mich & Leaf & Crushing & Oral & 10 \\
\hline \multirow[t]{2}{*}{$\begin{array}{l}\text { Malva parviflora } \\
\text { L. (Malvaceae) }\end{array}$} & Zebenya & $\begin{array}{l}\text { AS-12- } \\
2017\end{array}$ & Herb & Diarrhea & Leaf & Pounding & Oral & 9 \\
\hline & & & & Mich & Leaf & Squeezing & Oral & 14 \\
\hline $\begin{array}{l}\text { Mangifera indica } \\
\text { L. (Anacardiaceae) }\end{array}$ & Mango & $\begin{array}{l}\text { AS-56- } \\
2017\end{array}$ & Tree & Mich & Leaf & Roasting & Dermal & 12 \\
\hline $\begin{array}{l}\text { Mentha } \times \text { piperita } \\
\text { L. (Lamiaceae) }\end{array}$ & Nana & $\begin{array}{l}\text { AS-40- } \\
2017\end{array}$ & Herb & Diarrhea & Leaf, stem & $\begin{array}{l}\text { Pounding after } \\
\text { mixing it with } \\
\text { Nigella sativa and } \\
\text { A. sativum }\end{array}$ & Oral & 8 \\
\hline $\begin{array}{l}\text { Moringa stenopetala } \\
\text { (Baker f) Cufod. } \\
\text { (Moringaceae) }\end{array}$ & Sheferaw & $\begin{array}{l}\text { AS-43- } \\
2017\end{array}$ & Tree & Hypertension & Leaf & $\begin{array}{l}\text { Drying and } \\
\text { Pounding, and } \\
\text { filtering it after } \\
\text { adding water }\end{array}$ & Oral & 6 \\
\hline $\begin{array}{l}\text { Musa } \times \text { paradisiaca } \\
\text { L. (Musaceae) }\end{array}$ & Muz & - & Shrub & Rough skin & Fruit & $\begin{array}{l}\text { Peeling to remove } \\
\text { the skin }\end{array}$ & Dermal & 10 \\
\hline $\begin{array}{l}\text { Myrsine africana } \\
\text { L. (Primulaceae) }\end{array}$ & Kechem & $\begin{array}{l}\text { AS-23- } \\
2017\end{array}$ & Shrub & Tapeworm & Fruit & Crushing & Oral & 4 \\
\hline $\begin{array}{l}\text { Nigella sativa } \\
\text { L. (Ranunculaceae) }\end{array}$ & $\begin{array}{l}\text { Tikur } \\
\text { Azmud }\end{array}$ & - & Herb & $\begin{array}{l}\text { Stomach } \\
\text { complaints }\end{array}$ & Seed & $\begin{array}{l}\text { Pounding it after } \\
\text { mixing it with } \\
\text { A. sativum, Ruta } \\
\text { chalepensis, and } \\
\text { A. cepa and then } \\
\text { adding lemon juice }\end{array}$ & Oral & 12 \\
\hline \multirow[t]{3}{*}{$\begin{array}{l}\text { Ocimum urticifolium } \\
\text { Roth (Lamiaceae) }\end{array}$} & Dema Kassie & $\begin{array}{l}\text { AS-42- } \\
2017\end{array}$ & Shrub & Mich & Leaf & $\begin{array}{l}\text { Boiling in water } \\
\text { after mixing it with } \\
\text { Withania } \\
\text { somnifera }\end{array}$ & Oral & 15 \\
\hline & & & & $\begin{array}{l}\text { Common } \\
\text { cold }\end{array}$ & Leaf & & $\begin{array}{c}\text { Inhale (oral and } \\
\text { nasal) }\end{array}$ & 8 \\
\hline & & & & Hypertension & Leaf & & $\begin{array}{c}\text { Inhale (oral and } \\
\text { nasal) }\end{array}$ & 6 \\
\hline \multirow{2}{*}{$\begin{array}{l}\text { Olea europaea } \\
\text { L. subsp. cuspidata } \\
\text { (Wall. \& G. Don) Cif. } \\
\text { (Oleaceae) }\end{array}$} & Weyra & $\begin{array}{l}\text { AS-49- } \\
2017\end{array}$ & Tree & $\begin{array}{l}\text { Uvula } \\
\text { infection }\end{array}$ & Leaf & $\begin{array}{l}\text { Pounding and then } \\
\text { chewing }\end{array}$ & Oral & 9 \\
\hline & & & & Wound & Leaf & Squeezing & $\begin{array}{l}\text { Dermal on the } \\
\text { infected part }\end{array}$ & 7 \\
\hline $\begin{array}{l}\text { Oxalis radicosa } \\
\text { A. Rich. (Oxalidaceae) }\end{array}$ & Shimburut & $\begin{array}{l}\text { AS-07- } \\
2017\end{array}$ & Herb & Snake bite & Root & Crushing & Oral & 8 \\
\hline
\end{tabular}


TABle 1: Continued.

\begin{tabular}{|c|c|c|c|c|c|c|c|c|}
\hline Scientific name & Local name & $\begin{array}{l}\text { Voucher } \\
\text { number }\end{array}$ & Habit & $\begin{array}{c}\text { Ailment } \\
\text { treated }\end{array}$ & $\begin{array}{c}\text { Plant part } \\
\text { used }\end{array}$ & $\begin{array}{c}\text { Preparation } \\
\text { methods }\end{array}$ & $\begin{array}{c}\text { Administration } \\
\text { route }\end{array}$ & $\begin{array}{c}\text { No. of } \\
\text { informants }\end{array}$ \\
\hline $\begin{array}{l}\text { Phytolacca } \\
\text { dodecandra L'Hér. } \\
\text { (Phytolaccaceae) }\end{array}$ & $\begin{array}{l}\text { Mehan } \\
\text { endod }\end{array}$ & $\begin{array}{l}\text { AS-25- } \\
2017\end{array}$ & Shrub & Snake bite & Root & Chewing & Oral & 4 \\
\hline $\begin{array}{l}\text { Plectranthus spp. } \\
\text { (Lamiaceae) }\end{array}$ & Tezeteza & $\begin{array}{l}\text { AS-20- } \\
2017\end{array}$ & Herb & Bleeding & Leaf & Crushing & Nasal (smelling) & 8 \\
\hline $\begin{array}{l}\text { Rhamnus prinoides } \\
\text { L'Hér. (Rhamnaceae) }\end{array}$ & Gesho & $\begin{array}{l}\text { AS-33- } \\
2017\end{array}$ & Shrub & Tonsillitis & Leaf & $\begin{array}{l}\text { Crushing or } \\
\text { chewing }\end{array}$ & Oral & 14 \\
\hline & & & & $\begin{array}{l}\text { Uvula } \\
\text { infection }\end{array}$ & Leaf & $\begin{array}{l}\text { Squeezing to } \\
\text { produce juice }\end{array}$ & Oral & 7 \\
\hline $\begin{array}{l}\text { Ricinus communis } \\
\text { L. (Euphorbiaceae) }\end{array}$ & Gulo & $\begin{array}{l}\text { AS-47- } \\
2017\end{array}$ & Shrub & Devil's illness & Leaf & $\begin{array}{l}\text { Pounding after } \\
\text { mixing it with } \\
\text { Withania } \\
\text { somnifera and } \\
\text { squeezing }\end{array}$ & Oral & 10 \\
\hline $\begin{array}{l}\text { Rumex abyssinicus } \\
\text { Jacq. (Polygonaceae) }\end{array}$ & Moqmoqo & - & Shrub & $\begin{array}{l}\text { Common } \\
\text { cold }\end{array}$ & Root & Burning & $\begin{array}{l}\text { Fumigation (oral } \\
\text { and nasal) }\end{array}$ & 15 \\
\hline $\begin{array}{l}\text { Rumex nervosus Vahl } \\
\text { (Polygonaceae) }\end{array}$ & Embacho & $\begin{array}{l}\text { AS-31- } \\
2017\end{array}$ & Shrub & Mental stress & Root & Boiling it in water & Dermal & 7 \\
\hline $\begin{array}{l}\text { Ruta chalepensis } \\
\text { L. (Rutaceae) }\end{array}$ & Tena Adam & $\begin{array}{l}\text { AS-32- } \\
2017\end{array}$ & Herb & Asthma & Leaf & $\begin{array}{l}\text { Adding it into a } \\
\text { boiled tea/coffee }\end{array}$ & Oral & 12 \\
\hline & & & & Stroke & Leaf/seed & $\begin{array}{l}\text { Pounding or } \\
\text { crushing }\end{array}$ & Oral & 3 \\
\hline & & & & Stomach ache & Leaf/stem & Crushing & Oral & 11 \\
\hline & & & & Devil's illness & Leaf & Unprocessed & Nasal (smelling) & 16 \\
\hline $\begin{array}{l}\text { Schinus molle } \\
\text { L. (Anacardiaceae) }\end{array}$ & $\begin{array}{l}\text { Qundo } \\
\text { berberie }\end{array}$ & $\begin{array}{l}\text { AS-60- } \\
2017\end{array}$ & Tree & Mich & Leaf & $\begin{array}{l}\text { Boiling it in water } \\
\text { after mixing it with } \\
\text { Psychotria sp. }\end{array}$ & $\begin{array}{l}\text { Inhalation (oral } \\
\text { and nasal) }\end{array}$ & 15 \\
\hline \multirow[t]{3}{*}{$\begin{array}{l}\text { Sida schimperiana } \\
\text { Hochst. ex A. Rich. } \\
\text { (Malvaceae) }\end{array}$} & Chifrig & $\begin{array}{l}\text { AS-05- } \\
2017\end{array}$ & Shrub & Toothache & Leaf & Grinding & Oral & 7 \\
\hline & & & & $\begin{array}{c}\text { Erectile } \\
\text { dysfunction }\end{array}$ & Root & $\begin{array}{l}\text { Mixing it with } \\
\text { Nigella sativa, } \\
\text { A. sativum, and } \\
\text { honey and boiling } \\
\text { it in water }\end{array}$ & Oral & 8 \\
\hline & & & & Evil eye & Root & Chewing & Oral & 9 \\
\hline $\begin{array}{l}\text { Silene macrosolen } \\
\text { A. Rich. } \\
\text { (Caryophyllaceae) }\end{array}$ & Wegert & - & Shrub & Devil's illness & Root & $\begin{array}{l}\text { Putting it on fire to } \\
\text { produce smoke }\end{array}$ & $\begin{array}{l}\text { Fumigation (oral } \\
\text { and nasal) }\end{array}$ & 8 \\
\hline \multirow[t]{2}{*}{$\begin{array}{l}\text { Solanum marginatum } \\
\text { L.f. (Solanaceae) }\end{array}$} & Embuay & $\begin{array}{l}\text { AS-02- } \\
2017\end{array}$ & Shrub & $\begin{array}{l}\text { Stomach } \\
\text { illness }\end{array}$ & Root & $\begin{array}{l}\text { Pounding by } \\
\text { mixing it with root } \\
\text { of Tragia sp. }\end{array}$ & Oral & 6 \\
\hline & & & & Snake bite & Root & Crushing & Oral & 8 \\
\hline $\begin{array}{l}\text { Terminalia brownii } \\
\text { Fresen. } \\
\text { (Combretaceae) }\end{array}$ & Inkoy & $\begin{array}{l}\text { AS-65- } \\
2017\end{array}$ & Shrub & $\begin{array}{l}\text { Uvula } \\
\text { infection }\end{array}$ & Leaf & Squeezing & Oral & 9 \\
\hline \multirow[t]{2}{*}{$\begin{array}{l}\text { Tragia spp. } \\
\text { (Euphorbiaceae) }\end{array}$} & Awl alit & $\begin{array}{l}\text { AS-11- } \\
2017\end{array}$ & Herb & Wound & Leaf & Crushing & Dermal & 9 \\
\hline & & & & Evil eye & Root & $\begin{array}{l}\text { Grinding or } \\
\text { pounding }\end{array}$ & Dermal & 6 \\
\hline \multirow[t]{2}{*}{$\begin{array}{l}\text { Trigonella foenum- } \\
\text { graecum L. (Fabaceae) }\end{array}$} & Abish & - & Herb & Evil eye & Leaf & $\begin{array}{l}\text { Crushing and } \\
\text { adding it on fire }\end{array}$ & $\begin{array}{l}\text { Fumigation } \\
\text { (nasal) }\end{array}$ & 10 \\
\hline & & & & Broken leg & Seed & $\begin{array}{l}\text { Grinding or } \\
\text { pounding or } \\
\text { boiling it in water }\end{array}$ & $\begin{array}{l}\text { Dermal on the } \\
\text { broken bone }\end{array}$ & 6 \\
\hline \multirow[t]{2}{*}{$\begin{array}{l}\text { Verbascum sinaiticum } \\
\text { Benth. } \\
\text { (Scrophulariaceae) }\end{array}$} & Yejib Chama & $\begin{array}{l}\text { AS-06- } \\
2017\end{array}$ & Herb & Mental stress & Root & $\begin{array}{l}\text { Squeezing and } \\
\text { boiling it in water }\end{array}$ & Dermal & 5 \\
\hline & & & & $\begin{array}{l}\text { Uterus } \\
\text { retention }\end{array}$ & Root & $\begin{array}{l}\text { Squeezing and } \\
\text { boiling it in water }\end{array}$ & Nasal & 7 \\
\hline
\end{tabular}


TABle 1: Continued.

\begin{tabular}{|c|c|c|c|c|c|c|c|c|}
\hline Scientific name & Local name & $\begin{array}{l}\text { Voucher } \\
\text { number }\end{array}$ & Habit & $\begin{array}{l}\text { Ailment } \\
\text { treated }\end{array}$ & $\begin{array}{c}\text { Plant part } \\
\text { used }\end{array}$ & $\begin{array}{c}\text { Preparation } \\
\text { methods }\end{array}$ & $\begin{array}{l}\text { Administration } \\
\text { route }\end{array}$ & $\begin{array}{c}\text { No. of } \\
\text { informants }\end{array}$ \\
\hline $\begin{array}{l}\text { Vicia faba } \\
\text { L. (Fabaceae) }\end{array}$ & Bakela & - & Herb & $\begin{array}{l}\text { Swelling on } \\
\text { skin }\end{array}$ & Seed & $\begin{array}{l}\text { Grinding or } \\
\text { pounding }\end{array}$ & Dermal & 15 \\
\hline \multirow{5}{*}{$\begin{array}{l}\text { Vitis vinifera Linn. } \\
\text { (Vitaceae) } \\
\text { Withania somnifera } \\
\text { (L.) Dunal } \\
\text { (Solanaceae) }\end{array}$} & & & & Cough & & Chewing & Oral & 10 \\
\hline & Wein & - & Tree & $\begin{array}{l}\text { Eye diseases } \\
\text { (dirt) }\end{array}$ & Seed & $\begin{array}{l}\text { Pounding and then } \\
\text { squeezing }\end{array}$ & Ocular & 5 \\
\hline & \multirow[t]{3}{*}{ Gizewa } & \multirow[t]{3}{*}{$\begin{array}{l}\text { AS-35- } \\
2017\end{array}$} & \multirow[t]{3}{*}{ Shrub } & Mich & Root & $\begin{array}{l}\text { Drying and } \\
\text { pounding }\end{array}$ & $\begin{array}{l}\text { Fumigation } \\
\quad \text { (nasal) }\end{array}$ & 12 \\
\hline & & & & & Root & Chewing & Oral & 8 \\
\hline & & & & Evil disease & Leaf & Boiling it in water & $\begin{array}{l}\text { Inhalation } \\
\text { (nasal) }\end{array}$ & 16 \\
\hline \multirow{3}{*}{$\begin{array}{l}\text { Zehneria scabra (L.f.) } \\
\text { Sond. (Cucurbitaceae) } \\
\text { Zingiber officinale } \\
\text { Roscoe } \\
\text { (Zingiberaceae) }\end{array}$} & Hareg resa & $\begin{array}{l}\text { AS-45- } \\
2017\end{array}$ & Herb & Mich & Leaf & Boiling it in water & $\begin{array}{l}\text { Inhalation (oral } \\
\text { and nasal) }\end{array}$ & 12 \\
\hline & \multirow[t]{2}{*}{ Zingible } & \multirow[t]{2}{*}{ - } & \multirow[t]{2}{*}{ Herb } & Tonsillitis & \multirow[t]{2}{*}{$\begin{array}{l}\text { Rhizome } \\
\text { (stem) }\end{array}$} & Chewing & Oral & 6 \\
\hline & & & & $\begin{array}{l}\text { Common } \\
\text { cold }\end{array}$ & & $\begin{array}{l}\text { Pounding and } \\
\text { boiling }\end{array}$ & $\begin{array}{c}\text { Inhale (oral, } \\
\text { nasal) }\end{array}$ & 15 \\
\hline \multirow[t]{2}{*}{$\begin{array}{l}\text { Ziziphus spina-christi } \\
\text { (Rhamnaceae) }\end{array}$} & \multirow[t]{2}{*}{ Qunqura } & \multirow[t]{2}{*}{$\begin{array}{l}\text { AS-52- } \\
2017\end{array}$} & \multirow[t]{2}{*}{ Tree } & Devil's illness & Leaf & $\begin{array}{l}\text { Pounding and then } \\
\text { squeezing }\end{array}$ & Oral & 7 \\
\hline & & & & Dandruff & Leaf & $\begin{array}{l}\text { Pounding after } \\
\text { adding water and } \\
\text { then squeezing }\end{array}$ & $\begin{array}{l}\text { Dermal on the } \\
\text { affected part }\end{array}$ & 13 \\
\hline
\end{tabular}

TABLE 2: Medicinal plants used to treat livestock ailments.

\begin{tabular}{|c|c|c|c|c|c|c|c|c|}
\hline Scientific name & $\begin{array}{l}\text { Local } \\
\text { name }\end{array}$ & $\begin{array}{l}\text { Voucher } \\
\text { number }\end{array}$ & Habit & $\begin{array}{l}\text { Ailment } \\
\text { treated }\end{array}$ & $\begin{array}{c}\text { Plant } \\
\text { part } \\
\text { used }\end{array}$ & $\begin{array}{l}\text { Preparation } \\
\text { method }\end{array}$ & $\begin{array}{l}\text { Administration } \\
\text { route }\end{array}$ & $\begin{array}{l}\text { No. of } \\
\text { informants }\end{array}$ \\
\hline \multirow[t]{2}{*}{ Acacia spp. (Fabaceae) } & Doret & $\begin{array}{c}\text { AS-28- } \\
2017\end{array}$ & Shrub & Wound & Leaf & Crushing & Dermal & 7 \\
\hline & & & & $\begin{array}{c}\text { Eye } \\
\text { infection }\end{array}$ & Leaf & Chewing & Ocular & 8 \\
\hline $\begin{array}{l}\text { Bidens prestinaria (Sch. Bip.) } \\
\text { Cufod. (Asteraceae) }\end{array}$ & Chigogot & $\begin{array}{l}\text { AS-18- } \\
2017\end{array}$ & Herb & Insect bite & Leaf & $\begin{array}{l}\text { Pounding to } \\
\text { produce juice }\end{array}$ & Dermal & 6 \\
\hline $\begin{array}{l}\text { Calpurnia aurea (Aiton) } \\
\text { Benth. (Fabaceae) }\end{array}$ & Digita & $\begin{array}{l}\text { AS-04- } \\
2017\end{array}$ & Herb & Mange & Leaf & Crushing & Dermal & 10 \\
\hline $\begin{array}{l}\text { Chenopodium ambrosioides } \\
\text { L. (Chenopodiaceae) }\end{array}$ & Sinign & $\begin{array}{l}\text { AS-09- } \\
2017\end{array}$ & Herb & $\begin{array}{l}\text { Chicken } \\
\text { flea }\end{array}$ & Root & Putting it on fire & $\begin{array}{l}\text { Fumigating the } \\
\text { house }\end{array}$ & 4 \\
\hline $\begin{array}{l}\text { Cissus quadrangularis } \\
\text { L. (Vitaceae) }\end{array}$ & Kimtita & $\begin{array}{c}\text { AS-44- } \\
2017\end{array}$ & Herb & Mange & $\begin{array}{l}\text { Whole } \\
\text { plant }\end{array}$ & Crushing & Dermal & 11 \\
\hline $\begin{array}{l}\text { Cyphostemma adenocaule } \\
\text { (Steud. ex A. Rich.) Desc. ex } \\
\text { Wild \& R.B. Drumm. } \\
\text { (Vitaceae) }\end{array}$ & $\begin{array}{c}\text { Aba } \\
\text { Woldu }\end{array}$ & $\begin{array}{l}\text { AS-10- } \\
2017\end{array}$ & Herb & $\begin{array}{l}\text { Leech } \\
\text { infestation }\end{array}$ & Root & Crushing & Oral & 13 \\
\hline $\begin{array}{l}\text { Dodonaea angustifolia L.f. } \\
\text { (Sapindaceae) }\end{array}$ & Kitkita & $\begin{array}{l}\text { AS-50- } \\
2017\end{array}$ & Shrub & $\begin{array}{c}\text { Bone } \\
\text { dislocation }\end{array}$ & Leaf & $\begin{array}{l}\text { Crushing and } \\
\text { pounding }\end{array}$ & Dermal & 4 \\
\hline $\begin{array}{l}\text { Echinops kebericho Mesfin } \\
\text { (Asteraceae) }\end{array}$ & Kebercho & - & Herb & Anthrax & Root & $\begin{array}{l}\text { Grinding to } \\
\text { yield juice }\end{array}$ & Oral & 10 \\
\hline Echinops spp. (Asteraceae) & Kushelie & $\begin{array}{c}\text { AS-27- } \\
2017\end{array}$ & Shrub & $\begin{array}{c}\text { Bone } \\
\text { dislocation }\end{array}$ & Root & Unprocessed & $\begin{array}{l}\text { Dermal (tie it on } \\
\text { the dislocated } \\
\text { bone) }\end{array}$ & 14 \\
\hline $\begin{array}{l}\text { Ficus vasta Forssk. } \\
\text { (Moraceae) }\end{array}$ & Warka & $\begin{array}{c}\text { AS-74- } \\
2017\end{array}$ & Tree & Thrips & $\begin{array}{l}\text { Stem } \\
\text { bark }\end{array}$ & $\begin{array}{l}\text { Pounding and } \\
\text { boiling it in } \\
\text { water }\end{array}$ & Oral & 7 \\
\hline $\begin{array}{l}\text { Kalanchoe marmorata Bak. } \\
\text { (Crassulaceae) }\end{array}$ & Endahul & $\begin{array}{l}\text { AS-01- } \\
2017\end{array}$ & Herb & Cancer & Root & Crushing & Dermal & 4 \\
\hline
\end{tabular}


TABle 2: Continued.

\begin{tabular}{|c|c|c|c|c|c|c|c|c|}
\hline Scientific name & $\begin{array}{l}\text { Local } \\
\text { name }\end{array}$ & $\begin{array}{l}\text { Voucher } \\
\text { number }\end{array}$ & Habit & $\begin{array}{l}\text { Ailment } \\
\text { treated }\end{array}$ & $\begin{array}{c}\text { Plant } \\
\text { part } \\
\text { used }\end{array}$ & $\begin{array}{l}\text { Preparation } \\
\text { method }\end{array}$ & $\begin{array}{l}\text { Administration } \\
\text { route }\end{array}$ & $\begin{array}{l}\text { No. of } \\
\text { informants }\end{array}$ \\
\hline $\begin{array}{l}\text { Lycopersicon esculentum } \\
\text { Mill. (Solanaceae) }\end{array}$ & Timatim & - & Herb & $\begin{array}{c}\text { Leech } \\
\text { infestation }\end{array}$ & Leaf & $\begin{array}{l}\text { Pounding after } \\
\text { adding water }\end{array}$ & Oral and nasal & 14 \\
\hline $\begin{array}{l}\text { Maytenus senegalensis (Lam.) } \\
\text { Exell (Celastraceae) }\end{array}$ & Qoqoba & $\begin{array}{l}\text { AS-71- } \\
2017\end{array}$ & Shrub & Insect bite & Leaf & $\begin{array}{l}\text { Crushing it to } \\
\text { yield juice }\end{array}$ & Dermal & 10 \\
\hline $\begin{array}{l}\text { Nicotiana tabacum } \\
\text { L. (Solanaceae) }\end{array}$ & Timbaho & $\begin{array}{l}\text { AS-15- } \\
2017\end{array}$ & Shrub & $\begin{array}{c}\text { Leech } \\
\text { infestation }\end{array}$ & Leaf & $\begin{array}{l}\text { Squeezing or } \\
\text { pounding after } \\
\text { adding water }\end{array}$ & Oral and nasal & 8 \\
\hline \multirow[t]{3}{*}{$\begin{array}{l}\text { Otostegia integrifolia Benth. } \\
\text { (Lamiaceae) }\end{array}$} & Tunjiut & $\begin{array}{l}\text { AS-68- } \\
2017\end{array}$ & Shrub & Wound & Leaf & Crushing & $\begin{array}{l}\text { Dermal on the } \\
\text { wound }\end{array}$ & 10 \\
\hline & & & & Ear disease & Leaf & $\begin{array}{l}\text { Pounding and } \\
\text { squeezing to } \\
\text { yield juice }\end{array}$ & Auricular & 7 \\
\hline & & & & $\begin{array}{c}\text { Flea } \\
\text { infestation }\end{array}$ & Leaf & Putting it on fire & Fumigating house & 7 \\
\hline $\begin{array}{l}\text { Portulaca oleracea } \\
\text { L. (Portulacaceae) }\end{array}$ & Antaria & $\begin{array}{l}\text { AS-63- } \\
2017\end{array}$ & Herb & $\begin{array}{c}\text { Bone } \\
\text { dislocation }\end{array}$ & Root & Crushing & $\begin{array}{l}\text { Dermal on the } \\
\text { dislocated bone }\end{array}$ & 10 \\
\hline $\begin{array}{l}\text { Ximenia americana } \\
\text { L. (Olacaceae) }\end{array}$ & Ikma & $\begin{array}{l}\text { AS-66- } \\
2017\end{array}$ & Shrub & Emaciation & $\begin{array}{l}\text { Stem } \\
\text { bark }\end{array}$ & $\begin{array}{l}\text { Boiling it in } \\
\text { water }\end{array}$ & Oral & 6 \\
\hline
\end{tabular}

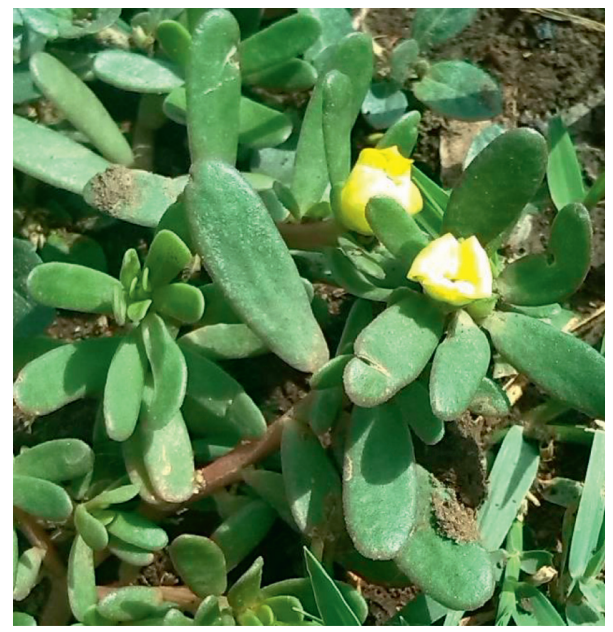

(a)

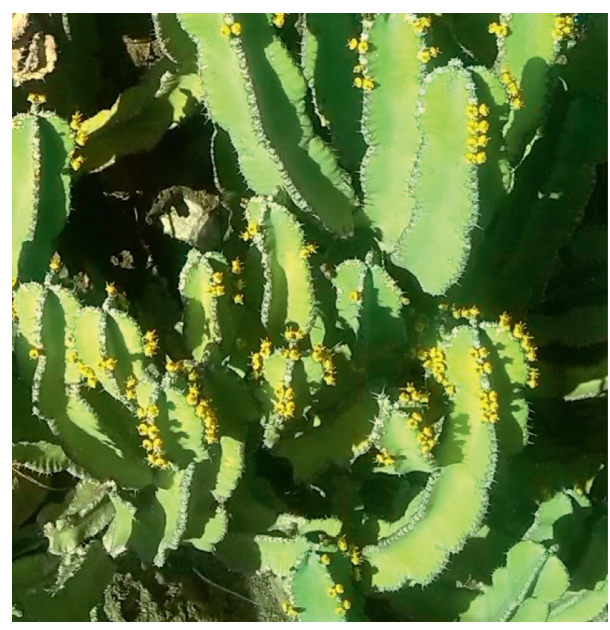

(c)

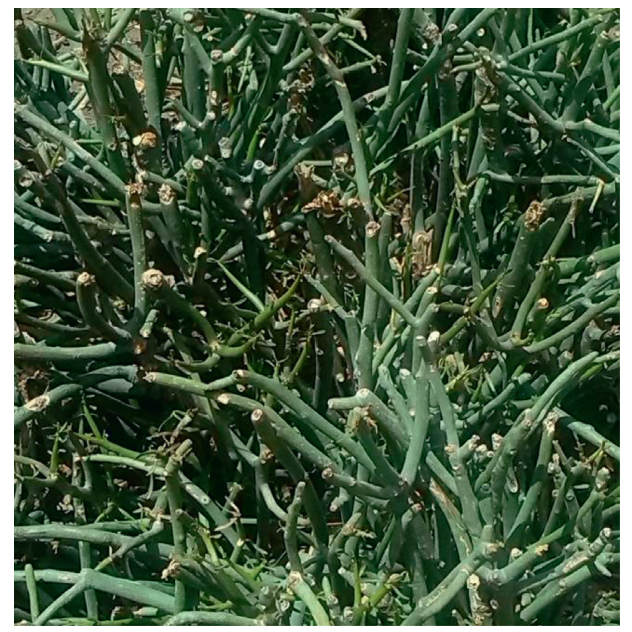

(b)

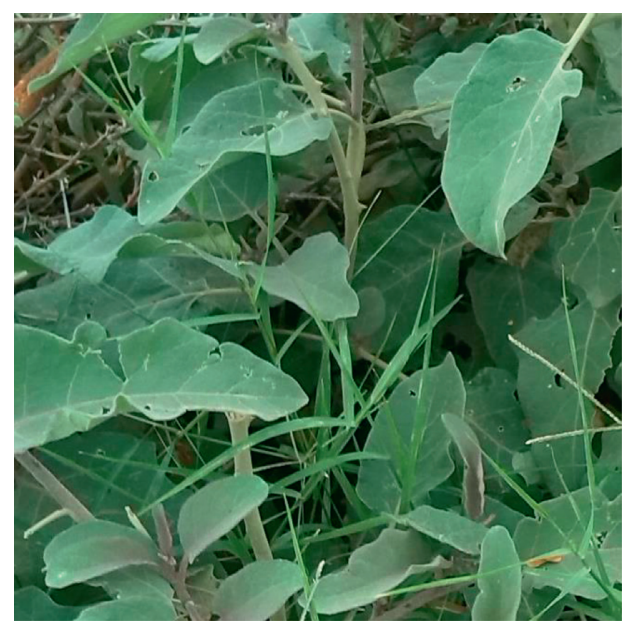

(d)

Figure 2: Continued. 


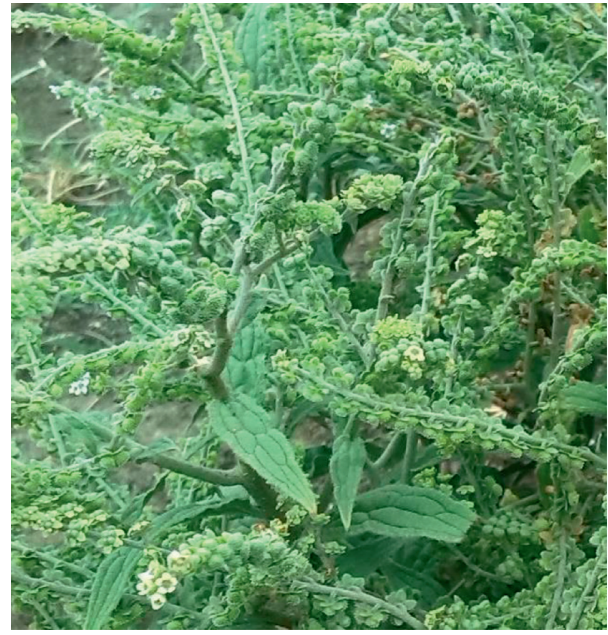

(e)

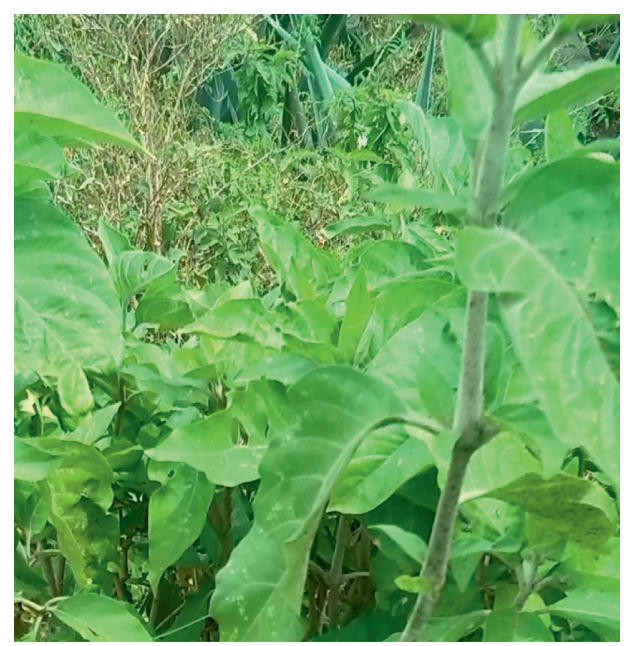

(f)

Figure 2: Some medicinal plants of Raya Kobo. (a) Antaria (Portulaca oleracea); (b) kinchibt (Euphorbia tirucalli); (c) qulqualda (Euphorbia spp.); (d) embuay (Cucumis dipsaceus); (e) yegid zemedie (Cynoglossum lanceolatum); and (f) simiza (Justicia schimperiana).

TABLE 3: Human diseases and the corresponding number of medicinal plants used for their treatments.

\begin{tabular}{|c|c|c|}
\hline SN & Ailment treated & No. of medicinal plants used \\
\hline 1 & Gastrointestinal complaints & 17 \\
\hline 2 & Mich (febrile illness) & 13 \\
\hline 3 & Wound & 10 \\
\hline 4 & Snake bite & 6 \\
\hline 5 & Devil's illness & 6 \\
\hline 6 & Mental stress & 5 \\
\hline 7 & Dislocated/broken bone & 5 \\
\hline 8 & Cough & 5 \\
\hline 9 & Evil eye & 5 \\
\hline 10 & Common cold & 4 \\
\hline 11 & Uvula infection & 4 \\
\hline 12 & Tinea nigra & 3 \\
\hline 13 & Jaundice & 3 \\
\hline 14 & Cancer & 3 \\
\hline 15 & Hemorrhoids & 2 \\
\hline 16 & Swelling on the skin & 2 \\
\hline 17 & Malaria & 2 \\
\hline 18 & Tonsillitis & 2 \\
\hline 19 & Wart & 2 \\
\hline 20 & Asthma & 2 \\
\hline 21 & Allergy & 1 \\
\hline 22 & Thorn inside the skin & 1 \\
\hline 23 & Toothache & 1 \\
\hline 24 & Hypertension & 1 \\
\hline 25 & Anthrax & 1 \\
\hline 26 & Hepatitis & 1 \\
\hline 27 & Headache & 1 \\
\hline 28 & Itch & 1 \\
\hline 29 & Bleeding & 1 \\
\hline 30 & Dandruff & 1 \\
\hline 31 & Erectile dysfunction & 1 \\
\hline 32 & Joint ache & 1 \\
\hline 33 & Black spider bite & 1 \\
\hline 34 & Eye disease & 1 \\
\hline 35 & Lung disease & 1 \\
\hline 36 & Rough skin & 1 \\
\hline 37 & Stroke & 1 \\
\hline 38 & Uterus retention & 1 \\
\hline
\end{tabular}


TABLE 4: Livestock diseases and the corresponding number of medicinal plants used for their treatments.

\begin{tabular}{lcc}
\hline SN & Ailment treated & No. of medicinal plants used \\
\hline 1 & Dislocated/broken bone & 3 \\
2 & Leech infestation & 3 \\
3 & Mange & 2 \\
4 & Insects bites & 2 \\
5 & Wound & 2 \\
6 & Anthrax & 1 \\
7 & Cancer & 1 \\
8 & Eye infection & 1 \\
9 & Thrips & 1 \\
10 & Flea infestation & 1 \\
11 & Ear disease & 1 \\
12 & Emaciation & 1 \\
\hline
\end{tabular}

importance in the study district [34]. Mich is a local term used by traditional medicine practitioners for human ailment mainly characterized by fever, headache, and sore lips but not well-understood and recognized by modern healthcare practitioners. The fact that relatively high number of medicinal plants is used for treating mich in the district could be an indication of the high health importance of the ailment and the lack of effective treatment against it in modern medicine.

Of the 17 medicinal plants used for treating livestock diseases, three medicinal plants were used to treat dislocated/broken bones and three more to treat leech infestation. Likewise, mange, insect bites, and wound are treated by two medicinal plants each; while the remaining seven ailments are treated by one medicinal plant each (Table 4). According to an unpublished 2019 local government data, mange and anthrax were reported as the top two of the eight diseases of veterinary importance in the district [34].

3.3. Plant Parts Used and Methods of Remedy Preparation and Administration. Leaves were the most commonly used plant parts in the preparation of remedies in Raya Kobo accounting for $53.1 \%$ of the total preparations, followed by roots $(21.8 \%)$ and fruits and seeds (12.9\%) (Figure 3). Ethnobotanical studies carried out elsewhere in Ethiopia $[20,38,40,45]$ also reported that leaves are the most widely used parts in the preparation of plant remedies. The wide use of leaves in the preparations of plant remedies may be attributed to the fact that leaves are much easier to process quickly as compared to other plant parts. Collection of leaves does not pose a great danger to the survival of individual plants as compared to the collection of underground parts, stems, and whole plants. Removal of up to $50 \%$ of leaves of plants does not significantly affect their growth [36]. Contrary to ours, studies conducted in other places of Ethiopia $[46,47]$ observed roots as the most widely used plant parts in preparing traditional remedies. Harvesting roots, if not performed carefully, may cause detrimental effects on the plants that could ultimately bring about their disappearance or extinction from their natural habitats $[6,48]$.

The preparation methods of plant remedies in the study district were diverse. The most commonly used methods

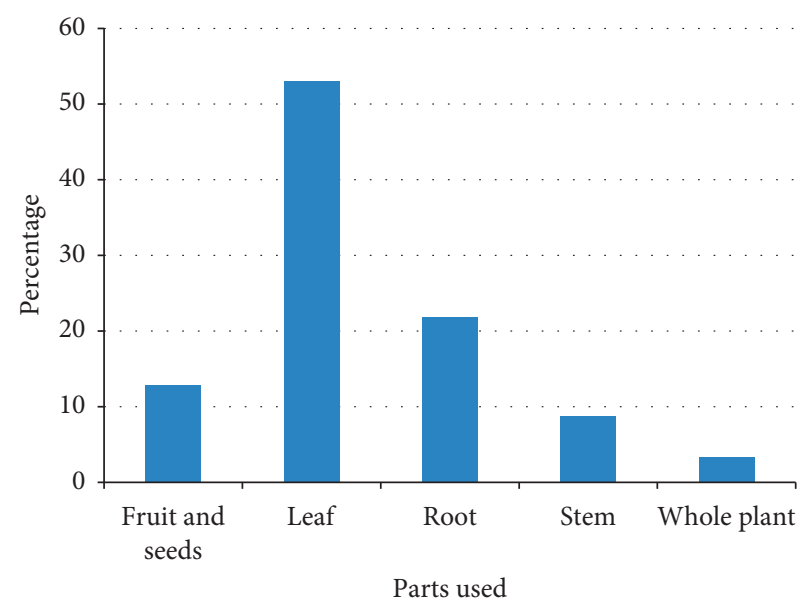

FIgURE 3: Proportions of plant parts used in preparation of remedies in Raya Kobo district.

Table 5: Remedy preparation methods in Raya Kobo district.

\begin{tabular}{lccc}
\hline SN & $\begin{array}{c}\text { Remedy preparation } \\
\text { method }\end{array}$ & $\begin{array}{c}\text { Number of } \\
\text { preparations }\end{array}$ & Percentage \\
\hline 1 & Grinding/pounding & 31 & 23.7 \\
2 & Crushing & 26 & 19.8 \\
3 & Boiling & 19 & 14.5 \\
4 & Chewing & 14 & 10.7 \\
5 & Squeezing & 11 & 8.4 \\
6 & Cutting and bleeding & 9 & 6.9 \\
7 & Pounding and squeezing & 4 & 3.1 \\
8 & Burning & 3 & 2.3 \\
9 & Squeezing and boiling & 2 & 1.5 \\
10 & Roasting and pounding & 2 & 1.5 \\
11 & Pounding and chewing & 2 & 1.5 \\
12 & Splitting and slicing & 2 & 1.5 \\
13 & Crushing and squeezing & 1 & 0.8 \\
14 & Roasting & 1 & 0.8 \\
15 & Crushing and burning & 1 & 0.8 \\
16 & Pounding and boiling & 1 & 0.8 \\
17 & Peeling & 1 & 0.8 \\
18 & Unprocessed & 1 & 0.8 \\
& Total & 131 & 100 \\
\hline
\end{tabular}

were grinding/pounding (23.7\%), crushing (19.8\%), boiling (14.5\%), chewing (10.7\%), squeezing $(8.4 \%)$, and cutting and bleeding (6.9\%) (Table 5). Similar studies conducted in the country by Girmay and Teshome [49], Amenu [40], and Hunde et al. [47] reported crushing, grinding, or pounding as the most common methods of preparation of plant remedies. But Lulekal et al. [46] reported boiling as the most frequently used method of remedy preparation in Mana Angetu district of Oromia State of Ethiopia. Choice of preparation methods may be influenced by the types and diversity of medicinal plants as well as the cultural background of the communities practicing traditional medicine.

Analysis of the routes of administration revealed that the majority of the plant remedies were given orally (53.7\%), followed by those applied dermally (or topically) (28.9\%) (Figure 4). Similar observations were reported by many 


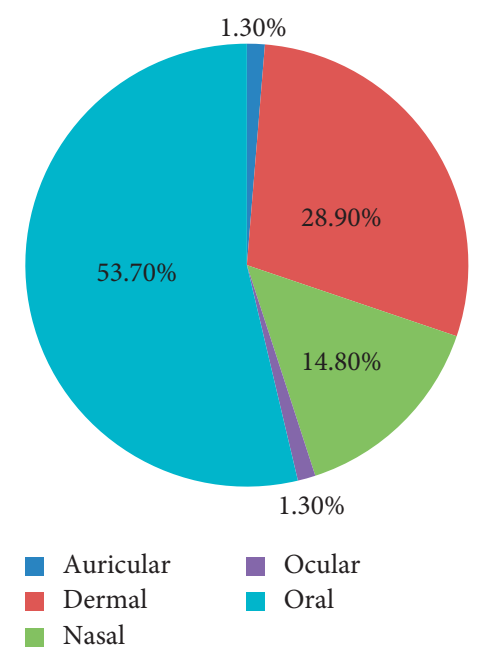

FIgURE 4: Frequency distribution of administration route of remedies in Raya Kobo district.

studies carried out in other parts of Ethiopia $[14,38,46,49-53]$. Ashagre [54] reported that both oral and dermal routes permit the quick physiological reaction of remedies to the causative agents of diseases to increase curative power. In the present study, informants affirmed that they advise their patients to be cautious during and after application of remedies depending on the types of ailments treated. For instance, in some cases, patients were restricted from drinking milk and local alcoholic drinks (e.g., tella and tej), eating meat and eggs, and having sexual intercourse as such practices are believed to interfere with the curative powers of the remedies.

3.4. Habitats and Abundance of and Threats to Medicinal Plants. Analysis of interview data revealed that majority $(52.2 \%)$ of the plants used in the traditional medicinal practices of the people in the study district were harvested only from the wild, while some are harvested from home gardens and cultivated fields (Table 6). Adefa and Getaneh [38], Adefa and Abraha [14], and Amenu [40] reported similar findings in their studies conducted in the Ethiopian districts of Chencha, Tehuledere, and Chelya, respectively. Tanto et al. [55] also reported that most Ethiopian medicinal plants are harvested from the wild. According to Mander et al. [56], 56,000 tons of medicinal plants are utilized in Ethiopia annually, of which $87 \%$ is obtained from the wild. Field observations made by the present investigators revealed that there was very little effort in Raya Kobo to conserve medicinal plants. Only few medicinal plants including Carica papaya, Ocimum urticifolium, and Rhamnus prinoides used in treating gastric problems, mich, and infection of uvula, respectively, were purposely grown in some home gardens or cultivated fields. These indicate that the medicinal flora in the study district is under big threat due to extensive environmental degradation. According to Leta [57], deforestation and overgrazing are serious problems in Raya Kobo and other north Ethiopian places.
TABle 6: Habitats of medicinal plants in Raya Kobo district.

\begin{tabular}{cccc}
\hline SN & Habit of the plants & $\begin{array}{c}\text { Number of medicinal } \\
\text { plants }\end{array}$ & Percentage \\
\hline 1 & Wild only & 48 & 52.70 \\
2 & Home garden only & 18 & 19.80 \\
3 & Cultivation field only & 16 & 17.60 \\
4 & Wild or home garden & 6 & 6.60 \\
5 & Wild or cultivation & 3 & 3.30 \\
& land & 91 & 100.0 \\
\hline
\end{tabular}

Of the total informants interviewed, majority (51.3\%) of them attested that most of the medicinal plants were either difficult or very difficult to find, while $48.7 \%$ of them claimed that most of the medicinal plants were easy to find in the immediate environment. According to them, deforestation (49.3\%), draught (22\%), and overgrazing (14\%) are the main factors for the depletion of medicinal plants in their places (Table 7). Most of the informants reported that they kept their knowledge of medicinal plants as secret with limited willingness to share to the younger generation. The limited interest of practitioners of traditional medicine in passing over their knowledge on medicinal plants to the younger generation is considered as another threat to the continuation of the practice in the study area. Other studies conducted elsewhere in the country also reported secrecy as a problem responsible for the loss of traditional medicinal knowledge and practices and the associated medicinal plants [20, 46, 48, 58].

Informants claimed that environmental protection/ rehabilitation $(60 \%)$, cultivation of medicinal plants in home gardens (14\%), reforestation (10\%), and demarcation of grazing lands (9.3\%) are the main measures that can help in the conservation of medicinal plants in the district (Table 8). They also revealed that there were few medicinal plants that were intentionally managed in home gardens and crop fields but primarily for other purposes such as food, fodder, spice, live fence, and shade. The investigators observed that there were efforts by some traditional medical practitioners to grow/cultivate medicinal plants, which were also used as sources of food and spices, in their home gardens and crop fields. According to Asfaw [59], of the medicinal plants managed in home gardens, only $6 \%$ is cultivated for medicinal uses only. The present investigators observed that annual reforestation programs in the study district mainly focused on exotic and few indigenous plants with very limited attention to plants of medicinal values.

3.5. Sources of Traditional Medicinal Knowledge. Informants of this study who have participated in the interviews claimed that they acquired knowledge of medicinal plants from different sources including family members (61.3\%), friends/acquaintances (14\%), and traditional healers (Table 9). A study carried out in Gimbi district, western Wollega zone of Ethiopia, reported that great majority of informants (91\%) cited parents as their sources 
Table 7: Reasons for depletion of medicinal plants in Raya Kobo district as reported by informants.

\begin{tabular}{lccc}
\hline SN & Reasons for depletion of medicinal plans & Number of informants & Percentage \\
\hline 1 & Deforestation & 74 & 49.3 \\
2 & Drought & 21 & 22.0 \\
3 & Over-grazing & 8 & 14.0 \\
4 & Overexploitation & 8 & 5.3 \\
5 & Firewood collection & 3 & 5.3 \\
6 & Environmental degradation & 3 & 2.0 \\
7 & Other factors & 150 & 2.0 \\
& Total & 100.0 \\
\hline
\end{tabular}

TABle 8: Ways of conserving medicinal plants in Raya Kobo district.

\begin{tabular}{lcc}
\hline SN & Conservation activity & Percentage \\
\hline 1 & Environmental protection/rehabilitation & 60.0 \\
2 & Cultivation in home gardens & 14.0 \\
3 & Reforestation & 10.0 \\
4 & Isolation of grazing lands & 9.3 \\
5 & Cultivation on agricultural plots & 5.3 \\
6 & Construction of check dams & 1.3 \\
& Total & 100.0 \\
\hline
\end{tabular}

TABLE 9: Sources of traditional knowledge on medicinal plants in Raya Kobo district.

\begin{tabular}{lccc}
\hline SN & Sources of knowledge & Frequency & Percentage \\
\hline 1 & Family members & 92 & 61.3 \\
2 & Friends/acquaintances & 21 & 14.0 \\
3 & Traditional healers & 17 & 11.3 \\
4 & Books & 7 & 4.7 \\
5 & Observation & 6 & 4.0 \\
6 & Relatives & 5 & 3.3 \\
7 & Modern health practitioners & 1 & 0.7 \\
8 & Others & 1 & 0.7 \\
& Total & 150 & 100.0 \\
\hline
\end{tabular}

of knowledge of traditional medicinal plants [60]. Other researchers have also reported similar findings elsewhere in Ethiopia [40, 61-65]. These observations imply that initiatives that promote the sharing of such knowledge have to be encouraged.

3.6. Acceptance of Medicinal Plant Remedies. This study revealed that treatment with medicinal plants was highly accepted in Raya Kobo because of the belief that medicinal plants were efficacious in managing different ailments. Most informants $(72.7 \%)$ reported that medicinal plants are more effective as compared to modern drugs. Some informants (14.7\%) claimed that there were ailments that could only be treated using medicinal plants and not with modern medications (Table 10). It is a well-known fact that traditional medicine is still recognized in different parts of the world as the preferred means to manage different ailments. Estimates show that about $80 \%$ of the Ethiopian population is still dependent on traditional medicine, which essentially involves the use of medicinal plants [4].

\subsection{Preference Ranking of Medicinal Plants Used for Treating} Mich. Analysis of preference ranking conducted on eight medicinal plants used for treating mich (febrile illness) in the study district revealed that Ocimum urticifolium is the most preferred plant, followed by Withania somnifera and Zehneria scabra (Table 11). Mich is an ailment in the district against which the highest number of medicinal plants was reported by the informants. Many other studies have showed that Ocimum urticifolium is widely used in Ethiopia for treating mich and other febrile illnesses [10, 48, 66-71]. A related species, Ocimum lamiifolium, is also widely employed in different parts of the country to treat mich and similar ailments $[6,10,13,72,73]$. The common use of Ocimum urticifolium and related species may imply the potency of species of the genus Ocimum in treating mich and febrile illnesses. Furthermore, studies conducted on medicinal properties of extracts of different Ocimum species showed their antipyretic properties [74-77]. Withania somnifera $[10,16,26,48,78]$ and Zehneria scabra $[8,16,24,26,79,80]$ are also widely reported to be effective in treating febrile-like illnesses.

3.8. Comparison of Knowledge of Medicinal Plants among Different Groups. Comparison of knowledge of medicinal plants of the respondents across ages-in terms of number of remedies they reported-showed a strong correlation $(n=150 ; r=0.709 ; p \leq 0.01)$ (Table 12$) ;$ as age increased, knowledge of medicinal plants increased. Moreover, comparisons among age groups in regard to their knowledge of medicinal plants using one-way ANOVA showed a statistically significant difference $(F=74.22 ; p \leq 0.05)$ implying that older informants have accumulated more knowledge and experience in the study district. This finding is in agreement with results of other ethnobotanical investigations carried out elsewhere in the country $[14,62,65,72]$. The low interest of the younger generation towards traditional medicine and medicinal plants may be influenced by the expansion of modern education and acculturation. On the other hand, results of studies by Yineger and Yewhalaw [45] and Adefa and Getaneh [38] have demonstrated the absence of correlation between the number of medicinal plant reported and the age of informants. 
TABLE 10: Reasons reported by informants in the district for acceptance of traditional medicinal plants.

\begin{tabular}{lccc}
\hline SN & Reasons for acceptance of medicinal plants & Frequency & Percentage \\
\hline 1 & Effectiveness & 109 & 72.7 \\
2 & Ailment not treated with modern medication & 22 & 8 \\
3 & Easy accessibility & 7 & 5.3 \\
4 & Cheaper cost & 2 & 4.7 \\
5 & Better efficacy than modern medication & 1 & 1.3 \\
6 & Absence of modern healthcare facility nearby & 1 & 0.7 \\
7 & Other reasons & 150 & 0.7 \\
& Total & 100 \\
\hline
\end{tabular}

Table 11: Preference ranking exercise conducted on eight plants used to treat mich in Raya Kobo district.

\begin{tabular}{|c|c|c|c|c|c|c|c|c|c|c|}
\hline \multirow{2}{*}{ SN } & \multirow{2}{*}{ Species } & \multicolumn{7}{|c|}{ Respondents } & \multirow{2}{*}{ Total } & \multirow{2}{*}{ Rank } \\
\hline & & 1 & 2 & 3 & 4 & 5 & 6 & 7 & & \\
\hline 1 & Azadirachta indica & 7 & 6 & 6 & 7 & 6 & 7 & 6 & 45 & 5 \\
\hline 2 & Cynoglossum lanceolatum & 5 & 6 & 4 & 6 & 7 & 4 & 6 & 38 & 8 \\
\hline 3 & Eucalyptus globulus & 6 & 7 & 8 & 7 & 7 & 5 & 7 & 47 & 4 \\
\hline 4 & Heliotropium cinerascens & 8 & 7 & 6 & 7 & 6 & 5 & 6 & 43 & 6 \\
\hline 5 & Justicia schimperiana & 6 & 7 & 5 & 7 & 7 & 6 & 7 & 41 & 7 \\
\hline 6 & Ocimum urticifolium & 8 & 7 & 8 & 8 & 8 & 7 & 8 & 54 & 1 \\
\hline 7 & Withania somnifera & 8 & 7 & 7 & 8 & 6 & 7 & 8 & 51 & 2 \\
\hline 8 & Zehneria scabra & 8 & 8 & 7 & 7 & 6 & 6 & 8 & 50 & 3 \\
\hline
\end{tabular}

TABLE 12: Correlations of informants' ages and number of medicinal plant citation.

\begin{tabular}{lccc}
\hline & & No. of plant citations & Age of informants \\
\hline & Pearson correlation & 1 & $0.709^{* *}$ \\
No. of plant citations & Significance (2-tailed) & 150 & 0.000 \\
& $N$ & $0.709^{* *}$ & 150 \\
Age of informants & Pearson correlation & 0.000 & 1 \\
& Significance (2-tailed) & 150 & 150 \\
\hline
\end{tabular}

** Correlation is significant at the 0.01 level (2-tailed).

However, there was no significance difference in the knowledge of medicinal plants expressed in terms of mean number of prescriptions of medicinal plants reported between men and women $(\mathrm{d} f=149 ; F=0.073 ; p>0.05)$. Men informants $(n=75)$ reported 617 prescriptions $($ mean $=8.2)$, while women informants $(n=75)$ reported 620 prescriptions (mean $=8.3$ ). Similar finding was reported by Alemayehu et al. [20] in a study conducted in Minjar-Shenkora district of the Amhara State of Ethiopia. Interview results of the present study also indicated that traditional knowledge was transferred within the family without special preference to either sex.

3.9. Status of Knowledge of Medicinal Plants in Raya Kobo. Of the 150 informants participated in the present study, 83 $(55.3 \%)$ rated themselves as having low level of knowledge of medicinal plants, while $59(39.3 \%)$ rated themselves as having medium level of knowledge. Only eight (5.4\%)
TABLE 13: Levels of education and medicinal plants knowledge reported by informants in Raya Kobo.

\begin{tabular}{lccccc}
\hline \multirow{2}{*}{ SN } & Educational & \multicolumn{4}{c}{ Knowledge of traditional } \\
& level & Low & Medium & High & \\
\hline 1 & Illiterate & $31(20.7 \%)$ & $20(13.3 \%)$ & $5(3.3 \%)$ & $56(37.3 \%)$ \\
2 & $\begin{array}{c}\text { Informal } \\
\text { education } \\
\text { Formal } \\
3\end{array}$ & $36(24.0 \%)$ & $23(15.3 \%)$ & $3(2.0 \%)$ & $62(41.3 \%)$ \\
& education & $16(10.7 \%)$ & $16(10.7 \%)$ & $0(0.0 \%)$ & $32(21.3 \%)$ \\
\hline & Total & $83(55.3 \%)$ & $59(39.3 \%)$ & $8(5.3 \%)$ & $150(100)$ \\
\hline
\end{tabular}

informants considered themselves as having high level of knowledge of medicinal plants (Table 13). Of the 56 illiterate informants participated in the study, 5 (9\%) of them reported that they have high level of knowledge of medicinal plants, 
and only 3 (5\%) of the 62 informants with informal education claimed to have high level of knowledge. However, none of the 32 informants with formal education claimed to have high level of knowledge of medicinal plants. This may be taken as an indication that the number of people with high level of knowledge of medicinal plants in the district is dwindling due to the influence of formal education, acculturation, and expansion of modern healthcare services.

\section{Conclusion}

The result of this study showed the existence of rich traditional knowledge in Raya Kobo district on the use of medicinal plants for treating many human and livestock ailments. Since herbal preparations are cheaper and are believed to be more effective, the majority of people in the district still give priority to visit traditional healers to seek treatments for themselves and for their families and livestock. Many of the plants used for traditional remedies are collected from the wild. The observation that majority of the remedies are prepared from leaves imply that ethnomedicinal and ethnoveterinary practices in the study area do not cause the depletion of plant biodiversity. Mich, the most common ailment in the study district, is treated or managed by the highest number of medicinal plants. The preference ranking conducted on eight medicinal plants used for treating this ailment showed that Ocimum urticifolium is the most preferred plant. These observations are important indications of the high potency of the plant against the disease to call for prioritized scientific investigation. Generally speaking, extensive documentation of ethnomedicinal and ethnoveterinary knowledge has to be pursued in the study district and neighboring regions before it is too late.

\section{Data Availability}

The data used to support this study are available from the corresponding author upon request.

\section{Ethical Approval}

The proposal was reviewed and approved by the Ethical Review Board (IRB) of Department of Biology, College of Natural and Computational Sciences, Mekelle University.

\section{Consent}

Oral informed consent was also obtained from people who participated in the study.

\section{Conflicts of Interest}

The authors declare that they have no conflicts of interest.

\section{Acknowledgments}

The authors express their gratitude to the informants for sharing their incredible traditional knowledge related to medicinal plants. The authors also thank Mr. Desale Alie and Mr. Abera Asefie for accompanying them while they were travelling within the study kebeles and their assistance in collecting and carrying plant specimens. The authors are very grateful to the administrative officials in Raya Kobo district for providing them with a support letter to travel freely in rural kebeles during the ethnobotanical survey and members of sector offices at the district for providing them with relevant information and documents concerning the study area. The authors are also grateful to Dr. Fesseha Abadi, Mekelle University, for assisting them in editing the manuscript and Mr. Nathan Dawit and Ms. Yordanos Desta for drawing the graphic abstract. Finally, the authors thank Mekelle University for funding the research.

\section{References}

[1] G. Bodeker, K. K. S. Bhat, J. Burley, and P. Vantomme, Wood Forest Products 11: Non-Medicinal Plants for Forest Conservation and Healthcare, Global Initiative for Traditional Systems (GIFTS) of Health, Food and Agriculture Organization of the United Nations, Rome, Italy, 1997.

[2] M. J. Balick and P. A. Cox, Plants, People, and Culture: The Science of Ethnobotany, Scientific American Library, New York, NY, USA, 1997.

[3] E. Kelbessa and S. Demissew, "Diversity of vascular plant taxa of the flora of Ethiopia and Eritrea," Ethiopian Journal of Biological Sciences, vol. 13, pp. 37-45, 2014.

[4] D. Abebe and E. Hagos, "Plants as a primary source of drugs in the traditional health practices of Ethiopia," in Plant Genetic Resources of Ethiopia, J. M. M. Engles, J. G. Hawkes, and M. Worede, Eds., pp. 1-13, Cambridge University Press, Cambridge, UK, 1991.

[5] D. Abebe and A. Ayehu, Medicinal Plants and Enigmatic Health Practices of Northern Ethiopia, B.S.P.E., Addis Ababa, Ethiopia, 1993.

[6] M. Giday, T. Teklehaymanot, A. Animut, and Y. Mekonnen, "Medicinal plants of the Shinasha, Agew-Awi and Amhara peoples in northwest Ethiopia," Journal of Ethnopharmacology, vol. 110, no. 3, pp. 516-525, 2007.

[7] M. Giday and G. Ameni, "An ethnobotanical survey on plants of veterinary importance in two woredas of Southern Tigray, Northern Ethiopia," SINET: Ethiopian Journal of Science, vol. 26, pp. 123-136, 2003.

[8] T. Gedif and H.-J. Hahn, "The use of medicinal plants in selfcare in rural central Ethiopia," Journal of Ethnopharmacology, vol. 87, no. 2-3, pp. 155-161, 2003.

[9] T. Teklehaymanot, M. Giday, G. Medhin, and Y. Mekonnen, "Knowledge and use of medicinal plants by people around Debre Libanos Monastery in Ethiopia," Journal of Ethnopharmacology, vol. 111, no. 2, pp. 271-283, 2007.

[10] T. Wondimu, Z. Asfaw, and E. Kelbessa, "Ethnobotanical study of medicinal plants around "Dheeraa" town, Arsi zone, Ethiopia," Journal of Ethnopharmacology, vol. 112, no. 1, pp. 152-161, 2007.

[11] J. Abbink, "Medicinal and ritual plants of the Ethiopian southwest: an account of recent research," Indigenous Knowledge and Development Monitor, vol. 3, pp. 6-8, 1995.

[12] M. Ragunathan and S. Mequanente, "Ethnomedicinal survey of folk drugs used in Bahirdar Zuria district, Northwestern Ethiopia," Indian Journal of Traditional Knowledge, vol. 8, pp. 281-284, 2009.

[13] T. Teklehaymanot, "Ethnobotanical study of knowledge and medicinal plants use by the people in Dek Island in Ethiopia," Journal of Ethnopharmacology, vol. 124, no. 1, pp. 69-78, 2009. 
[14] M. Adefa and B. Abraha, "Ethnobotanical survey of traditional medicinal plants in Tehuledere district, south Wollo, Ethiopia," Journal Medicinal Plant Research, vol. 5, pp. 6233-6242, 2011.

[15] Z. Birhanu, "Traditional use of medicinal plants by the ethnic groups of Gondar Zuria district, north-western Ethiopia," Journal of Natural Medicines, vol. 13, pp. 46-53, 2013.

[16] E. Lulekal, Z. Asfaw, E. Kelbessa, and P. van Damme, "Ethnomedicinal study of plants used for human ailments in Ankober district, north Shewa zone, Amhara region, Ethiopia," Journal of Ethnobiology and Ethnomedicine, vol. 9, pp. 1-13, 2013.

[17] G. Gebeyehu, Z. Asfaw, A. Enyew, and N. Raja, "Ethnobotanical study of traditionally used medicinal plants and their conservation status in Mecha Wereda, West Gojjam zone of Ethiopia," International Journal of Public Health Research, vol. 2, pp. 137-154, 2014.

[18] D. Kalu and A. Seid, "Ethnobotanical study of medicinal plants in Ankober Woreda, central Ethiopia," Ethiopian Journal of Science and Technology, vol. 7, pp. 105-114, 2014.

[19] E. Lulekal, Z. Asfaw, E. Kelbessa, and P. van Damme, "Ethnoveterinary plants of Ankober district, north Shewa zone, Amhara region, Ethiopia," Journal of Ethnobiology Ethnomedicine, vol. 10, pp. 1-19, 2014.

[20] G. Alemayehu, Z. Asfaw, and E. Kelbessa, "Plant diversity and ethnobotany in Berehet district, north Shewa zone of Amhara region (Ethiopia) with emphasis on wild edible plants," Journal of Medicinal Plants Studies, vol. 3, pp. 93-105, 2015.

[21] T. Mekuanent, A. Zebene, and Z. Solomon, "Ethnobotanical study of medicinal plants in Chilga district, northwestern Ethiopia," Journal of Natural Remedies, vol. 15, no. 2, pp. 88-112, 2015.

[22] Y. Limenih, S. Umer, and M. Wolde-Mariam, "Ethnobotanical study on traditional medicinal plants in Dega Damot Woreda, Amhara region, north Ethiopia," International Journal of Research in Pharmaceutical Sciences, vol. 5, pp. 258-273, 2015.

[23] Z. Getnet, S. Chandrodyam, and G. Masresha, "Studies on traditional medicinal plants in ambagiorgis area of Wogera district, Amhara regional state, Ethiopia," International Journal of Pure and Applied Biosciences, vol. 4, pp. 38-45, 2016.

[24] M. Meragiaw, Z. Asfaw, and M. Argaw, "The status of ethnobotanical knowledge of medicinal plants and the impacts of resettlement in Delanta, northwestern Wello, northern Ethiopia," Evidence-Based Complementary and Alternative Medicine, vol. 2016, Article ID 5060247, 24 pages, 2016.

[25] Y. Simegniew, S. Leshe, Y. Abebe, and N. Minuye, "Ethnobotanical study of medicinal plants used to treat human diseases in Enarj Enawga district, east Gojjam Zone, Amhara region, Ethiopia," SM Journal of Medicinal Plant Studies, vol. 1, p. 1006, 2017.

[26] G. Chekole, "Ethnobotanical study of medicinal plants used against human ailments in Gubalafto district, northern Ethiopia," Journal of Ethnobiology and Ethnomedicine, vol. 13, pp. 1-29, 2017.

[27] N. Amsalu, Y. Bezie, M. Fentahun, A. Alemayehu, and G. Amsalu, "Use and conservation of medicinal plants by indigenous people of Gozamin Wereda, east Gojjam zone of Amhara region, Ethiopia: an ethnobotanical approach," Evidence-Based Complementary and Alternative Medicine, vol. 2018, Article ID 2973513, 23 pages, 2018.

[28] T. Kelemu and W. Wolde, "Ethnobotanical study of indigenous knowledge on medicinal plants used to treat diseases in selected districts of Amhara regional state, Ethiopia," Journal of Medicinal Plants Research, vol. 12, pp. 528-536, 2018.

[29] S. W. Yohannis, Z. Asfaw, and Kelbessa, "Ethnobotanical study of medicinal plants used by local people in Menz Gera Midir district, north Shewa zone, Amhara regional state, Ethiopia," Journal of Medicinal Plants Research, vol. 12, pp. 296-314, 2018.

[30] Raya Kobo District Agricultural and Rural Development Office, Unpublished Government 2016 Agriculture and Rural Development Data, Raya Kobo, Kobo, Ethiopia, 2016.

[31] A. Adugna, "Amhara demography and health," 2018, http:// www.ethiodemographyandhealth.org/.

[32] CSA (Central Statistics Agency of Ethiopia), Human Population Census of Ethiopia Central Statistical Agency of Ethiopia, CSA, Addis Ababa, Ethiopia, 2007.

[33] Raya Kobo District Agricultural and Rural Development Office, Unpublished Government 2019 Agriculture and Rural Development Data, Raya Kobo, Kobo, Ethiopia, 2019.

[34] Raya Kobo District Health Office, Common Communicable Diseases in the Raya Kobo District, Unpublished Document, Raya Kobo Health Office, Raya Kobo, Kobo, Ethiopia, 2019.

[35] G. J. Martin, Ethnobotany: A Method Manual, Chapman \& Hall, London, UK, 1995.

[36] M. Poffenberger, B. McGean, A. Khare, and J. Campbell, Field Method Manual, Volume II. Community Forest Economy and Use Pattern: Participatory and Rural Appraisal (PRA) Methods in South Gujarat, India, Society for Promotion of Wastelands Development, New Delhi, India, 1992.

[37] M. N. Alexiades, "Collecting ethnobotanical data: an introduction to basic concepts and techniques," in Selected Guidelines for Ethnobotanical Research: A Field Manual, M. N. Alexiades, Ed., pp. 52-94, The New York Botanical Garden, New York, NY, USA, 1996.

[38] M. Adefa and S. Getaneh, "Medicinal plants biodiversity and local healthcare management system in Chencha district; Gamo Gofa, Ethiopia," Journal of Pharmacognosy Phytotherapy, vol. 2, pp. 284-293, 2013.

[39] D. Hunde, "Use and management of traditional plants by indigenous people of Boosat district Welenchiti area," M.Sc. thesis, Addis Ababa University, Addis Ababa, Ethiopia, 2001.

[40] E. Amenu, "Use and management of medicinal plants by indigenous people of Ejaji area (Chelya Wereda) west Shewa, Ethiopia: an ethnobotanical approach," M.Sc. thesis, Addis Ababa University, Addis Ababa, Ethiopia, 2007.

[41] M. Thulin, "Fabaceae, in flora of Ethiopia," in Volume 3: Pittosporaceae to Araliaceae, I. Hedberg and S. Edwards, Eds., pp. 49-251, The National Herbarium, Addis Ababa University, Addis Ababa, Ethiopia, 1989.

[42] M. G. Gilbert, "Euphorbiaceae, in flora of Ethiopia and Eritrea," in Volume 2, Part 2: Canellaceae to Euphorbiaceae, S. Edwards, M. Tadesse, and I. Hedberg, Eds., pp. 265-380, The National Herbarium, Addis Ababa University, Addis Ababa, Ethiopia, 1995.

[43] M. Tadesse, "Flora of Ethiopia," Volume 4, Part 2: Asteraceae (Compositae), The National Herbarium, Addis Ababa University, Addis Ababa, Ethiopia, 2004.

[44] O. Ryding, "Lamiaceae," in Flora of Ethiopia and Eritrea. Volume 5: Gentianaceae to Cyclocheilaceae, pp. 516-604, The National Herbarium, Addis Ababa University, Addis Ababa, Ethiopia, 2006.

[45] H. Yineger and D. Yewhalaw, "Traditional medicinal plant knowledge and use by local healers in Sekoru district, Jimma zone, southwestern Ethiopia," Journal of Ethnobiology and Ethnomedicine, vol. 3, pp. 1-7, 2007. 
[46] E. Lulekal, E. Kelbessa, T. Bekele, and H. Yineger, "An ethnobotanical study of medicinal plants in Mana Angetu district, southeastern Ethiopia," Journal of Ethnobiology and Ethnomedicine, vol. 4, pp. 1-10, 2008.

[47] H. Hunde, Z. Asfaw, and E. Kelbessa, "Use of traditional medicinal plants by people of Boosat subdistrict, central eastern Ethiopia," Ethiopian Journal Health Sciences, vol. 16, pp. 141-155, 2006.

[48] M. Maryo, S. Nemomissa, and T. Bekele, "An ethnobotanical study of medicinal plants of the Kembatta ethnic group in Enset-based agricultural landscape of Kembatta Tembaro," Asian Journal of Plant Science Research, vol. 5, pp. 142-161, 2015.

[49] T. Girmay and Z. Teshome, "Assessment of traditional medicinal plants used to treat human and livestock ailments and their threatening factors in Gulomekeda district, Northern Ethiopia," International Journal of Emerging Trends in Science and Technology, vol. 4, no. 4, pp. 5061-5070, 2017.

[50] D. Abebe, "Traditional medicine in Ethiopia: the attempts being made to pro- mote it for effective and better utilization," SINET: Ethiopian Journal of Science, vol. 9, pp. 61-69, 1986.

[51] D. Hunde, Z. Asfaw, and E. Kelbessa, "Use and management of ethno veterinary medicinal plants of indigenous people in (Boosat) Wolenchit area Ethiopia," Journal of Biological Sciences, vol. 3, pp. 113-132, 2004.

[52] K. Balemie, E. Kelbessa, and Z. Asfaw, "Indigenous medicinal plants utilization, management and threats in Fentalle area, eastern Shewa," Ethiopian Journal of Biological Sciences, vol. 3, pp. 37-58, 2004.

[53] G. Yirga, M. Teferi, and M. Kasaye, "Survey of medicinal plants used to treat human ailments in Hawzen district, northern Ethiopia," International Journal of Biodiversity and Conservation, vol. 3, pp. 709-714, 2011.

[54] M. Ashagre, "Ethnobotanical study of medicinal plants in guji agro-pastoralists, bule hora district of borana Zone, Oromia region, Ethiopia," M.Sc. thesis, Addis Ababa University, Addis Ababa, Ethiopia, 2011.

[55] T. Tanto, M. Giday, N. Aklilu, and T. Hunduma, Medicinal Plants Biodiversity, National Biodiversity Strategy and Action Plan (NBSAP) Project, Institute of Biodiversity Conservation and Research, Addis Ababa, Ethiopia, 2003.

[56] M. Mander, B. Emana, Z. Asfaw, and B. Busa, Marketing of Medicinal Plants in Ethiopia: A Survey of the Trade in Medicinal Plants, Institute of Biodiversity Conservation and Research, Addis Ababa, Ethiopia, 2006.

[57] E. F. Leta, "The link between food security and land degradation: analysis of determinants in drought prone areas of Northeast Ethiopia. A case of Sekota Woreda," M.Sc. thesis, Addis Ababa University, Addis Ababa, Ethiopia, 2008.

[58] D. Hunde, C. Abdeta, T. Berhan, and M. Sharma, "Medicinal plants use and conservation practices in Jimma zone," International Journal of Biodiversity and Conservation, vol. 7, pp. 202-210, 2015.

[59] Z. Asfaw, "The role of home gardens in the production and conservation of medicinal plants," in Biodiversity Conservation and Sustainable Use of Medicinal Plants in Ethiopia, M. Zewdu and A. Demissie, Eds., pp. 76-91, Institute of Biodiversity Conservation and Research, Addis Ababa, Ethiopia, 2001.

[60] E. Tolassa, "Use and conservation of traditional medicinal plants by indigenous people in Gimbi Woreda, western Wellega, Ethiopia," M.Sc. thesis, Addis Ababa University, Addis Ababa, Ethiopia, 2007.
[61] T. Gedif and H. Hahn, "Herbalists in Addis Ababa and Butajira, central Ethiopia: mode of service delivery and traditional pharmaceutical practice," Ethiopian Journal of Health Development, vol. 16, pp. 191-197, 2002.

[62] M. Giday, Z. Asfaw, T. Elmqvist, and Z. Woldu, "An ethnobotanical study of medicinal plants used by the Zay people in Ethiopia," Journal of Ethnopharmacology, vol. 85, no. 1, pp. 43-52, 2003.

[63] K. Deribe, A. Amberbir, B. Getachew, and Y. Mussema, "A historical overview of traditional medicine practices and policy in Ethiopia," Ethiopian Journal of Health Development, vol. 20, pp. 127-134, 2006.

[64] Z. Birhanu, A. Endale, and Z. Shewamene, "An ethnomedicinal investigation of plants used by traditional healers of Gondar town, north-western Ethiopia," Journal of Medicinal Plants Studies, vol. 3, pp. 36-43, 2015.

[65] T. Teklehaymanot, "An ethnobotanical survey of medicinal and edible plants of Yalo Woreda in Afar regional state, Ethiopia," Journal of Ethnobiology and Ethnomedicine, vol. 13, pp. 1-26, 2017.

[66] F. Gashe and F. Worku, "Ethnopharmacological survey of medicinal plants used for the treatment of human diseases in Abay-Chomen district, Eastern Wollega zone, western Ethiopia," Ethiopian Journal of Health Sciences, vol. 2, pp. 131-146, 2007.

[67] T. Flatie, T. Gedif, K. Asres, and T. Gebremariam, "Ethnomedical survey of Berta ethnic group Assosa zone, Benishangul-Gumuz regional state, midwest Ethiopia," Journal of Ethnobiology and Ethnomedicine, vol. 5, pp. 1-11, 2009.

[68] M. Megersa, Z. Asfaw, E. Kelbessa, A. Beyene, and B. Woldeab, "An ethnobotanical study of medicinal plants in Wayu Tuka district, east Welega zone of Oromia regional state, west Ethiopia," Journal of Ethnobiology and Ethnomedicine, vol. 9, no. 1, p. 68, 2013.

[69] B. Kidane, T. van Andel, L. van der Maesen, and Z. Asfaw, "Use and management of traditional medicinal plants by Maale and Ari ethnic communities in Southern Ethiopia," Journal of Ethnobiology and Ethnomedicine, vol. 10, no. 1, p. 46, 2014.

[70] G. Chekole, Z. Asfaw, and E. Kelbessa, "Ethnobotanical study of medicinal plants in the environs of Tara-Gedam and Amba Remnant forests of libo Kemkem district, northwest Ethiopia," Journal of Ethnobiology and Ethnomedicine, vol. 11, no. 1, p. 4, 2015.

[71] M. Kebebew, "Diversity, knowledge and use of medicinal plants in Abay Chomen district, Horo Guduru Wollega zone, Oromia region of Ethiopia," Journal of Medicinal Plants Research, vol. 11, pp. 480-500, 2007.

[72] M. Giday, Z. Asfaw, and Z. Woldu, "Medicinal plants of the Meinit ethnic group of Ethiopia: an ethnobotanical study," Journal of Ethnopharmacology, vol. 124, pp. 513-521, 2009.

[73] A. Belayneh and N. F. Bussa, "Ethnomedicinal plants used to treat human ailments in the prehistoric place of Harla and Dengego valleys, Eastern Ethiopia," Journal of Ethnobiology and Ethnomedicine, vol. 10, pp. 1-17, 2014.

[74] S. Godhwani, J. L. Godhwani, and D. S. Vyas, "Ocimum sanctum: an experimental study evaluating its anti-inflammatory, analgesic and antipyretic activity in animals," Journal of Ethnopharmacology, vol. 21, no. 2, pp. 153-163, 1987.

[75] E. Vyas, A. Debella, L. Zerihun, D. Abebe, and F. Teka, "Antipyretic properties of the aqueous and ethanol extracts of the leaves of Ocimum suave and Ocimum lamiifolium in mice," Journal of Ethnopharmacology, vol. 88, no. 1, pp. 85-91, 2003. 
[76] G. I. Gege-Adebayo, A. S. Bassi, V. U. Igbokwe, and M. O. Shafe, "Antipyretic effect of Ocimum gratissium on Brewer's yeast induced fever in Wistar rats," Journal of Medicine and Medical Sciences, vol. 4, pp. 247-251, 2013.

[77] V. Balakrishna, S. Pamu, and D. Pawar, "Evaluation of antipyretic activity of Ocimum sanctum Linnusing Brewer's yeast induced pyrexia in albino rats," Journal of Indian Posthodontic Society, vol. 1, pp. 55-58, 2017.

[78] B. Abera, "Medicinal plants used in traditional medicine by Oromo people, Ghimbi district, southwest Ethiopia," Journal of Ethnobiology and Ethnomedicine, vol. 10, pp. 1-15, 2014.

[79] K. Karunamoorthi and E. Tsehaye, "Ethnomedicinal knowledge, belief and self-reported practice of local inhabitants on traditional antimalarial plants and phytotherapy," Journal of Ethnopharmacology, vol. 141, no. 1, pp. 143-150, 2012.

[80] S. Suleman and T. Alemu, "A survey on utilization of ethnomedicinal plants in Nekemte town, east Wellega (Oromia), Ethiopia," Journal of Herbs, Spices \& Medicinal Plants, vol. 18, no. 1, pp. 34-57, 2012. 